Pure Appl. Chem., Vol. 85, No. 7, pp. 1515-1609, 2013.

http://dx.doi.org/10.1351/PAC-REC-06-04-06

(c) 2013 IUPAC, Publication date (Web): 6 June 2013

\title{
Definitions of terms relating to mass spectrometry (IUPAC Recommendations 2013)*
}

\author{
Kermit K. Murray ${ }^{1, \ddagger}$, Robert K. Boyd ${ }^{2}$, Marcos N. Eberlin³, \\ G. John Langley ${ }^{4}$, Liang $\mathrm{Li}^{5}$, and Yasuhide Naito ${ }^{6}$ \\ ${ }^{1}$ Department of Chemistry, Louisiana State University, Baton Rouge, LA, USA; \\ ${ }^{2}$ Institute for National Measurement Standards, National Research Council, \\ Ottawa, Ontario, Canada; ${ }^{3}$ Department of Chemistry, University of Campinas, \\ Campinas, Brazil; ${ }^{4}$ Chemistry, Faculty of Natural and Environmental Sciences, \\ University of Southampton, Southampton, UK; ${ }^{5}$ Department of Chemistry, \\ University of Alberta, Edmonton, Alberta, Canada; ${ }^{6}$ Graduate School for the \\ Creation of New Photonics Industries, Hamamatsu, Japan
}

\begin{abstract}
This document contains recommendations for terminology in mass spectrometry. Development of standard terms dates back to 1974 when the IUPAC Commission on Analytical Nomenclature issued recommendations on mass spectrometry terms and definitions. In 1978, the IUPAC Commission on Molecular Structure and Spectroscopy updated and extended the recommendations and made further recommendations regarding symbols, acronyms, and abbreviations. The IUPAC Physical Chemistry Division Commission on Molecular Structure and Spectroscopy's Subcommittee on Mass Spectroscopy revised the recommended terms in 1991 and appended terms relating to vacuum technology. Some additional terms related to tandem mass spectrometry were added in 1993 and accelerator mass spectrometry in 1994. Owing to the rapid expansion of the field in the intervening years, particularly in mass spectrometry of biomolecules, a further revision of the recommendations has become necessary. This document contains a comprehensive revision of mass spectrometry terminology that represents the current consensus of the mass spectrometry community.
\end{abstract}

Keywords: definitions; glossary; IUPAC Analytical Chemistry Division; mass spectrometry; terms.

\section{INTRODUCTION}

Mass spectrometry is the study of matter through the formation of gas-phase ions that are detected and characterized by their mass and charge. The recommendations in this document pertain to fundamental, technical, and experimental aspects of ion formation, mass selection, detection, and analysis of mass spectra.

In 1991, the Subcommittee on Mass Spectroscopy of the IUPAC Physical Chemistry Division Commission on Molecular Structure issued recommendations on mass spectrometry terminology $[1,2]$. These recommendations were supplemented by terms, prepared for the Analytical Chemistry Division, related to tandem mass spectrometry of organic molecules (IUPAC Commission on Microchemical

\footnotetext{
*Sponsoring body: IUPAC Analytical Chemistry Division: see more details on page 1600.

¥Corresponding author: E-mail: kkmurray@1su.edu
} 
Techniques and Trace Analysis and the Working Group on Organic Trace Analysis [3]) and accelerator mass spectrometry (IUPAC Commission on Radiochemistry and Nuclear Techniques [4]) and were included as a chapter in the Compendium of Analytical Nomenclature, known as the "Orange Book" [5]. Many of these terms are included in the Compendium of Chemical Terminology, known as the "Gold Book" [6]. Values for the physical constants and their symbols appearing in the Orange and Gold Books are taken from Quantities, Units and Symbols in Physical Chemistry, also known as the "Green Book" [7].

Over the years, IUPAC and the American Society for Mass Spectrometry (ASMS) have both worked in developing mass spectrometry terminology. The ASMS Measurements and Standards Committee published its Standard Definitions of Terms Related to Mass Spectrometry in 1991 [8]; the document represented the compilation of terms and definitions assembled between 1984 and 1990 by the Measurements and Standards Committee and closely paralleled the IUPAC terminology effort. A list of terms was presented at the ASMS Conference in Palm Springs, California in June of 1997 by the Measurements and Standards Committee and was available on the ASMS website between 1998 and 2000 [9]. There have been several other notable efforts at developing mass spectrometry terminology. A book that includes an extensive critical assessment of mass spectrometry terminology, Mass Spectrometry Desk Reference, was published in 2000 with a second edition in 2006 [10]. Another book, entitled A Dictionary of Mass Spectrometry, was published in 2010 [11]. The most recent peer-reviewed document is a glossary of terms for separations coupled to mass spectrometry that was published in the Journal of Chromatography A in 2010 [12].

During the nearly two decades since the last recommendations of IUPAC and ASMS, the field of mass spectrometry has changed radically. The introduction of soft ionization methods for the analysis of biological molecules has expanded the scope of mass spectrometry from its early roots in the analysis of inorganic and organic species into other fields such as biology and medicine. The expansion in scope has led to a growth in the number of terms and also in the use of those terms by those outside the field of mass spectrometry. As a result, this compilation of terms and definitions represents an extensive revision and expansion of the previous compilation. All defined terms recommended here are referenced to peer-reviewed literature.

\section{CONTROVERSIAL TERMS}

In the nearly two decades since the last recommendations on mass spectrometry terminology, there has been tremendous growth and expansion and, in some cases, divergence in opinion on correct usage. Below is a discussion of the rationale for the recommendations made for some of the more controversial terms.

\section{Labeling of mass spectra}

The labeling of the $x$-axis of a mass spectrum engendered the most discussion during the creation of this document; however, in spite of a general desire for a better way to label the $x$-axis of mass spectra, there was no broad consensus for any of the proposed changes. Therefore, this document continues the use of the definitions of the Gold Book [6] and the similar definitions in the Orange Book [5]. The Gold Book recommendation is for the use of $\mathrm{m} / \mathrm{z}$ as an abbreviation for mass-to-charge ratio, a dimensionless quantity obtained by dividing the mass number of an ion by its charge number [6].

The thomson unit, defined as the quotient of mass in units of $u$ and the number of charges $(z)$, was proposed nearly two decades ago [13], but has not been widely adopted and is therefore not recommended. Labeling the $x$-axis of a mass spectrum with any unit of mass such as dalton (Da), atomic mass unit (amu), or unified atomic mass unit (u) is strongly discouraged due to the confusion that would result when reporting spectra of multiply charged ions. The quantity plotted on the $x$-axis of a mass spectrum is a function of both the mass and charge of the ion. Furthermore, the use of amu in place of 
$\mathrm{u}$ is strongly discouraged in all cases; it has been used to denote atomic masses measured relative to the mass of a single atom of ${ }^{16} \mathrm{O}$, or to the isotope-averaged mass of an oxygen atom, or to the mass of a single atom of ${ }^{12} \mathrm{C}$.

\section{Resolution and resolving power}

The IUPAC definition of resolution in mass spectrometry expresses this value as $m / \Delta m$, where $m$ is the mass of the ion of interest and $\Delta m$ is the peak width (peak width definition) or the spacing between two equal intensity peaks with a valley between them no more than $10 \%$ of their height $(10 \%$ valley definition) [6]. Resolving power in mass spectrometry is defined as the ability of an instrument or measurement procedure to distinguish between two peaks at $\mathrm{m} / \mathrm{z}$ values differing by a small amount and expressed as the peak width in mass units [6]. Mass resolving power is defined separately as $m / \Delta m$ in a manner similar to that given above for mass resolution [6]. These definitions of mass resolving power and resolving power in mass spectrometry are contradictory, the former is expressed as a dimensionless ratio and the latter as a mass. The definitions for resolution in mass spectrometry and resolving power in mass spectrometry come from Todd's 1991 recommendations [1], and the definition for mass resolving power comes from Beynon's 1978 recommendations [14]. Beynon's work contains no definition for mass resolution.

Alternative definitions for resolution and resolving power in mass spectrometry have been proposed $[10,15]$. It has been suggested that resolution be given by $\Delta m$ and resolving power by $m / \Delta m$; however, these definitions are not widely used.

The majority of the mass spectrometry community uses resolution as defined by IUPAC. The term resolving power is not widely used as a synonym for resolution. In this document, the IUPAC definition of resolution in mass spectrometry remains in place. The definition of resolving power has been adapted from the current IUPAC definition of mass resolving power.

\section{Collision-induced dissociation (CID) and collisionally activated dissociation (CAD)}

The terms collision-induced dissociation (CID) and collisionally activated dissociation $(C A D)$ are both recommended by IUPAC [6] and are used interchangeably in recent literature. They are listed as synonyms in this document.

\section{Neutral species}

Although the word "neutral" is commonly used as an adjective, it is often used in mass spectrometry as a noun to indicate an atom or molecule as in the reaction of an ion with a neutral. The terms "neutral" and "neutral species" are used interchangeably in this document. This recommendation differs from the current recommendation for ion/neutral species reaction that recommends against using "neutral" as a noun [6].

\section{Ions in fragmentation reactions}

The anthropomorphic terms for ions involved in fragmentation reactions, for example, "daughter ion", have fallen into disuse after strong sentiments against the use of the term were voiced two decades ago $[16,17]$. The term product ion is recommended in place of daughter ion and precursor ion in place of parent ion. The use of $n^{\text {th }}$-generation product ion is recommended in place of granddaughter ion and similar terms. 


\section{Abbreviations of combined methods}

The hyphen, or alternatively the slash (forward stroke), can be used to indicate combined methods such as gas chromatography separation combined with mass spectrometry detection. Thus, the above combination can be written as gas chromatography-mass spectrometry or alternatively as gas chromatography/mass spectrometry. The corresponding abbreviations are GC-MS or GC/MS. The first use of a hyphen to indicate the combination of a separation method with mass spectrometry was in the early 1960s [18], and the use of a slash separator was in the 1970s [19]. The term "hyphenated techniques" was coined in 1980 [20]. Currently, hyphens and slashes are used interchangeably [12]. The journal Rapid Communications in Mass Spectrometry has in the past recommended that the combination of two analytical techniques be designated by a slash [21]. A recent Journal of Chromatography glossary also favors this usage [12]. IUPAC recommends that hyphens be used to describe variants of separation techniques, for example, gas-liquid chromatography and pyrolysis-gas chromatography [6]. The authors of this document are evenly split in their preference for hyphen or slash. For consistency with the prior recommendations, we use the hyphen for combined techniques but note that the slash can be used interchangeably.

\section{Ionization method terminology}

There have been recent rapid developments in ionization methods such as ambient mass spectrometry and laser desorption ionization. This has led to a proliferation of names and acronyms, not all of which have widespread use. We have limited the terminology related to ionization techniques that have been in existence for more than 5 years and have been reported in over 100 publications from multiple research groups.

\section{Isotope delta}

The relative difference of isotope values, also known as the isotope delta, is the subject of some controversy regarding acceptable units. It is often expressed as parts per thousand (ppt) or parts per million (ppm), both of which are deprecated [7].

\section{A NOTE REGARDING NEW AND REVISED TERMS}

Terms that can be found in the Orange Book [5] and the Gold Book [6] are indicated by "from [5]" and "from [6]", respectively. Terms that have been revised are indicated by "revised from [5]" or "revised from [6]". Rewording and additional cross-referencing are not considered as revision in this document. New terms are not identified other than by lack of such notation. Deprecated terms and synonyms are included as separate entries with a note directing the reader to the appropriate term. Terms appearing elsewhere in the document are indicated by italic type when first mentioned in a definition, and related terms are indicated where appropriate.

\section{DOCUMENT ORGANIZATION}

Previous compilations of mass spectrometry terms have been organized alphabetically within sections. This document is organized in glossary format: alphabetically with no sections. It is not intended to be encyclopedic, but rather a list of terms with brief definitions supported by references to publications in the peer-reviewed literature. 


\section{1. absolute quantitation of proteins (AQUA)}

Method for absolute quantitation of proteins via measurements on constituent proteolytic peptides using chemically synthesized isotope-labeled peptides as surrogate internal standards for mass spectrometry. From [22].

\section{2. abundance sensitivity}

Ratio of the maximum ion current recorded at a specified $\mathrm{m} / \mathrm{z}$ value to the maximum ion current arising from the same species recorded at a neighboring $\mathrm{m} / \mathrm{z}$ value.

Note: The abundance sensitivity is a measure of the contribution of the peak "tail" of a major isotope (with a certain $\mathrm{m} / \mathrm{z}$ value) to an adjacent $\mathrm{m} / \mathrm{z}$ value which in some cases might more than $1 \mathrm{~m} / \mathrm{z}$ removed. Its value is dependent on the resolving power of the mass spectrometer.

Revised from [5,6] using additional information from [23].

\section{3. accelerating potential}

Electrical potential difference used to impart translational energy to ions.

Note: Accelerating voltage is often used as a synonym, but the terms are equivalent only in the case of a static accelerating potential.

From [24].

\section{4. accelerating voltage}

This term is deprecated.

See accelerating potential.

\section{5. accelerator mass spectrometry (AMS)}

Mass spectrometry technique in which atoms and molecules from a sample are ionized, accelerated to $\mathrm{MeV}$ energies and separated according to their momentum, charge, and energy, allowing high discrimination for measurement of isotope abundances.

From [4].

\section{6. accurate mass}

Experimentally determined mass of an ion of known charge.

Note 1: Can be used to determine elemental composition to within limits defined by both the accuracy and precision of the measurement.

Note 2: Accurate mass and exact mass are not synonymous. Accurate mass refers to a measured mass, and exact mass refers to a calculated mass.

See also exact mass.

From $[10,25]$. 


\section{7. accurate mass tag}

Value of the mass of a peptide from a protein that has been chemically or enzymatically cleaved into fragments. The mass is recorded with sufficiently high accuracy and precision that it can be used in identification of the protein.

See also peptide mass fingerprinting $(P M F)$.

From $[26,27]$.

\section{8. acyl cation}

See acylium ion .

\section{9. acylium ion} acyl cation

Even-electron ion of general formula $\mathrm{RC} \mathrm{O}^{+}$(or its resonance form $\mathrm{R}-\mathrm{C}^{+}=\mathrm{O}$ ). From [6].

\section{0. adiabatic ionization}

Removal of an electron from an atom, radical, or molecule in its lowest energy state, thus producing an ion in its lowest energy state.

From $[5,6]$.

\section{1. adduct ion}

Ion formed by the interaction of a precursor ion with one or more atoms or molecules to form an ion containing all the constituent atoms of the precursor ion as well as the additional atoms from the associated atoms or molecules.

Note: For example, a $\mathrm{Na}^{+}$adduct of a molecule (M) that is represented as $[\mathrm{M}+\mathrm{Na}]^{+}$. See also anionized molecule, cationized molecule, protonated molecule.

From $[5,6]$.

\section{2. after mass analysis}

This term is obsolete.

Term used to describe the total ion current measured as the sum of all the separate ion currents carried by the different ions of the same sign contributing to the mass spectrum.

See also before mass analysis.

From $[5,6]$.

\section{3. a-ion}

Fragment ion containing the peptide N-terminus formed upon dissociation of a peptide ion at the peptide backbone $\mathrm{C}-\mathrm{C}$ bond.

From [28]. 


\section{4. $\alpha$-cleavage}

Homolytic cleavage where the bond fission occurs between the atom adjacent to the atom at the apparent charge site and an atom removed from the apparent charge site by two bonds.

Note: In the case of a distonic ion, it is important to distinguish between fragmentation adjaSee also $\beta$-cleavage. cent to the charge site and fragmentation adjacent to the radical site.

From $[5,6]$

\section{5. alkylideneaminylium ion}

See iminylium ion.

\section{6. alkyl cation}

Even-electron positively charged saturated hydrocarbon ion containing no aromatic groups, a special case of carbenium ions.

From $[29,30]$.

\section{7. all-glass heated inlet system (AGHIS)}

All-glass inlet system for ion sources operating under vacuum that can be heated to about $500{ }^{\circ} \mathrm{C}$ and allows low vapor pressure materials to be introduced into the mass spectrometer without breaking vacuum.

From [31].

\section{8. allyl cation}

Even-electron ion formulated as $\mathrm{R}^{1} \mathrm{R}^{2} \mathrm{C}=\mathrm{CR}^{3} \mathrm{C}^{+} \mathrm{R}^{4} \mathrm{R}^{5}$ and its resonance forms. From $[32,33]$.

\section{9. ambient ionization}

Desorption of molecules and formation of ions outside the mass spectrometer directly from samples in their native environment with no or minimal sample preparation. Examples are desorption electrospray ionization (DESI) and direct analysis in real time (DART).

Note: "Direct analysis in real time" is a proprietary term indicating the formation of ions from a solid or liquid sample at atmospheric pressure through the interaction of a gas stream containing internally excited atoms or molecules with the surface. The excited-state species are typically formed in a glow discharge in helium or nitrogen. The term should

From [34]. only be used to describe the commercial product.

\section{0. ambient mass spectrometry}

Mass spectrometry analysis performed using an ambient ionization technique. From [34]. 


\section{1. analyte}

Component of a sample being analyzed; typically a specific chemical compound. Revised from [5,6] using additional information from [35,36].

\section{2. angle resolved mass spectrometry}

Technique used to investigate details of the collisional excitation process by studying trends relating scattering angle of the precursor ion, internal energy transferred, and the masses of the ion and its collision partner.

From [37].

\section{3. anionized molecule}

Ion formed by the association of an anion with a molecule, $\mathrm{M}$.

Note 1: For example, $[\mathrm{M}+\mathrm{Cl}]^{-}$.

Note 2: The terms quasi-molecular ion and pseudo-molecular ion are deprecated and should not be used in place of anionized molecule.

See also adduct ion.

From [38].

\section{4. appearance energy (AE)}

Deprecated: appearance potential.

Minimum energy that must be imparted to an atom or molecule to produce a detectable amount of a specified ion. In electron ionization mass spectrometry, minimum electron energy necessary for the detection of a given fragment ion.

Note: This experimental quantity can vary with the detection sensitivity of the instrument Revised from $[5,6]$. used, reflecting different kinetic shifts.

\section{5. appearance potential}

This term is deprecated.

See appearance energy.

\section{6. array detector}

Detector comprising several ion collection elements, arranged in a line or grid where each element is an individual detector.

From $[39,40]$.

\section{7. association reaction associative ion/molecule reaction}

Reaction of a (slow-moving) ion with a neutral in which the reactants combine to form a single ionized species with no further dissociation.

Revised from [5,6] using additional information from [41]. 


\section{8. associative ionization}

Ionization process in which two atoms or molecules, one or both of which is in an excited state, react to form a single positive ion and an electron.

See also dissociative ionization, Penning ionization.

From $[5,6]$.

\section{9. associative ion/molecule reaction}

See association reaction.

\section{0. atmospheric pressure ionization (API)}

Ionization process in which ions are formed from atoms or molecules at atmospheric pressure.

Note: Atmospheric pressure ionization is not a synonym for atmospheric pressure chemical ionization.

Revised from [6] using additional information from [42,43].

\section{1. atmospheric pressure chemical ionization (APCI)}

Chemical ionization of a sample that is a gas or nebulized liquid, using an atmospheric pressure corona discharge or beta emitter such as ${ }^{63} \mathrm{Ni}$.

From [43].

\section{2. atmospheric pressure matrix-assisted laser desorption/ionization (AP MALDI)}

Matrix-assisted laser desorption/ionization in which the sample target is at atmospheric pressure. From [44].

\section{3. atmospheric pressure photoionization (APPI)}

Direct ionization of molecules at atmospheric pressure by electron detachment induced by photons forming $\mathrm{M}^{+\bullet}$, or atmospheric pressure chemical ionization in which the reactant ions are generated by photoionization of suitable dopant species and subsequent ion/molecule reactions of their molecular ions.

From [45].

\section{4. atomic mass unit, amu}

See unified atomic mass unit.

Revised from [6] using additional information from [46].

\section{5. autodetachment}

Process whereby a negative ion in a discrete state with energy greater than the detachment threshold loses an electron spontaneously without further interaction with an energy source.

See also autoionization.

From [47]. 


\section{6. autoionization}

Formation of an ion when an atom or molecule in a discrete state with an internal energy greater than the ionization threshold loses an electron spontaneously without further interaction with an energy source.

See also autodetachment.

Revised from [5] using additional information from [48].

\section{7. auxiliary gas}

Gas used in a spray ion source in addition to the nebulizing gas to aid in solvent removal. From $[49,50]$.

\section{8. average mass}

Mass of an ion or molecule weighted for its isotopic composition.

See also monoisotopic mass, nominal mass.

Revised from [5] using additional information from [51].

\section{9. axial ejection}

Ejection of an ion from an ion trap in a direction parallel to the longitudinal axis of the trap. From [52].

\section{0. axialization}

Technique used in Fourier-transform ion cyclotron resonance mass spectrometers to limit the translational motion of the trapped ions so that they occupy a small volume near the trap center, thus optimizing performance criteria including resolving power.

From $[53,54]$.

\section{1. background mass spectrum}

Mass spectrum observed when no analyte is introduced into the mass spectrometer.

See also residual spectrum.

Revised from [5,6] using additional information from [11].

\section{2. base peak (BP)}

Peak in a mass spectrum that has the greatest intensity.

Note: $\quad$ This term may be applied to the mass spectra of pure substances or mixtures.

From $[5,6]$.

\section{3. base peak chromatogram (BPC) base peak ion chromatogram}

Chromatogram obtained by plotting the signal of the ions represented by the base peak detected in each of a series of mass spectra recorded as a function of retention time. 
Note: This term should be used for samples introduced following a chromatographic separation.

From [55].

\section{4. base peak ion chromatogram}

See base peak chromatogram (BPC).

\section{5. bath gas}

See buffer gas.

\section{6. beam mass spectrometer}

Mass spectrometer in which an ion beam accelerated from the ion source is transmitted through a $\mathrm{m} / \mathrm{z}$ analyzer, or analyzers, to the detector.

From [56].

\section{7. before mass analysis}

This term is obsolete.

Total ion current measured as the sum of all the separate ion currents for ions of the same sign prior to mass analysis.

See also after mass analysis.

From $[5,6]$.

\section{8. benzyl ion}

Even-electron ion $\mathrm{C}_{7} \mathrm{H}_{7}{ }^{+}$with the same carbon skeleton as toluene that has lost a hydrogen atom from the methyl side-chain that carries the positive charge.

See also tropylium ion, tolyl ion.

From [57].

\section{9. $\beta$-cleavage}

Homolytic cleavage where the bond fission occurs between an atom removed from the apparent charge site atom by two bonds and an atom adjacent to that atom and removed from the apparent charge site by three bonds.

See also $\alpha$-cleavage.

From $[5,6]$.

\section{0. b-ion}

Fragment ion containing the peptide N-terminus formed upon dissociation of a peptide ion at the peptide backbone $\mathrm{C}-\mathrm{N}$ bond.

From [28]. 


\section{1. blackbody infrared radiative dissociation (BIRD)}

Special case of infrared multiphoton dissociation wherein excitation of the reactant ion is caused by absorption of infrared photons radiating from heated blackbody surroundings, which are usually the walls of a vacuum chamber.

See also infrared multiphoton dissociation (IRMPD).

From [58].

\section{2. bottom-up proteomics}

Method of protein identification that uses proteolytic digestion before analysis by liquid chromatography and mass spectrometry. Proteins can be isolated by gel electrophoresis prior to digestion or, in shotgun proteomics, the protein mixture is digested and the resulting peptides are separated by liquid chromatography.

See also top-down proteomics.

From $[59,60]$.

\section{Brubaker lens Brubaker pre-filter}

Additional set of four short, cylindrical electrodes, mounted co-linearly with a transmission quadrupole mass spectrometer that is excited with some fraction of the AC potential but not the DC potential applied to the main quadrupole. The Brubaker lens thus acts as a high-pass filter for ion $\mathrm{m} / \mathrm{z}$, but the resulting delay in application of the DC potential component also results in a reduction in defocusing by fringe fields and thus significantly improves overall ion transmission efficiency at a given mass resolution.

From [61].

\section{Brubaker pre-filter}

See Brubaker lens.

\section{5. buffer gas \\ bath gas}

Inert gas used for collisional reduction of the internal or translational energy of ions, such as in ion traps.

See also collision gas.

From [62].

\section{6. capillary exit fragmentation}

This term is deprecated.

See in-source collision-induced dissociation. 


\section{7. carbanion}

Even-electron anion with a significant portion of the excess negative charge located on a carbon atom that usually has three substituent groups and an unshared pair of electrons for a total of eight valence electrons, with general formula $\mathrm{R}^{1} \mathrm{R}^{2} \mathrm{R}^{3} \mathrm{C}^{-}$where the $\mathrm{R}$ groups are any organic structure.

From [6].

\section{8. carbenium ion}

Carbocation that has at least one important contributing structure containing a tervalent carbon atom with a vacant p-orbital. Common examples are even-electron hypovalent ions with the charge formally localized on a carbon atom, with general formula $\mathrm{R}^{1} \mathrm{R}^{2} \mathrm{R}^{3} \mathrm{C}^{+}$where the $\mathrm{R}$ groups are any monovalent organic structure.

Note: In earlier literature, such ions were referred to as carbonium ions. Both types are collectively referred to as carbocations.

Revised from [6].

\section{9. carbocation}

Cation containing an even number of electrons with the excess positive charge formally located on a carbon atom. This is a general term embracing carbenium ions, carbonium ions, and cations derived from corresponding carbon-centred free radicals by removal of the unpaired electron.

Note: Such carbocations may be named by adding the word "cation" to the name of the corresponding radical, but such names do not imply structure (e.g., whether three- or fiveFrom $[5,6]$. coordinated carbon atoms are present).

\section{0. carbonium ion}

Even-electron hypervalent carbocation in which the charge is located on a carbon atom that carries a fifth covalent bond (e.g., $\mathrm{H}_{5} \mathrm{C}^{+}$), the most abundant reagent ion formed in chemical ionization using methane as reagent gas.

Note: This term was formerly used to describe ions of the type now referred to as carbenium ions.

From [6].

\section{1. cationized molecule}

Ion formed by the association of a cation with a molecule $\mathrm{M}$.

Note 1: For example, $[\mathrm{M}+\mathrm{Na}]^{+},[\mathrm{M}+\mathrm{K}]^{+}$, and $\left[\mathrm{M}+\mathrm{NH}_{4}\right]^{+}$.

Note 2: The terms quasi-molecular ion and pseudo-molecular ion are deprecated and should not See also adduct ion. be used in place of cationized molecule.

From [62]. 


\section{2. centroid acquisition}

Procedure of recording mass spectra in which an automated computer-based system detects peaks, calculates the centroid based on the average $\mathrm{m} / \mathrm{z}$ value weighted by the intensity, and assigns $\mathrm{m} / \mathrm{z}$, values based on a calibration file. Only the centroid $\mathrm{m} / \mathrm{z}$ value and the peak magnitude are stored.

See also profile mode.

From [63].

\section{3. channel electron multiplier (CEM)}

See continuous dynode particle multiplier.

\section{4. channel electron multiplier array (CEMA)}

See microchannel plate.

\section{5. charged residue model}

Theoretical model for production of highly charged macromolecules by electrospray ionization in which the excess charges on electrosprayed droplets are transferred to and remain on molecules enclosed within the droplets after solvent evaporation.

See also ion evaporation model.

From [64].

\section{6. charge exchange ionization (CEI)}

Interaction of an ion with an atom or molecule in which the charge on the ion is transferred to the neutral species without the dissociation of either species.

From $[5,6]$.

\section{7. charge exchange reaction}

See charge-transfer reaction (in mass spectrometry).

\section{8. charge inversion mass spectrum}

Plot of the relative abundances of ions that result from a charge inversion reaction as a function of $m / z$. From $[5,6]$.

\section{9. charge inversion reaction}

Reaction of an ion with a neutral, surface, or ion of opposite polarity, in which the charge on the product ion is reversed in sign with respect to the reactant ion.

See also charge inversion mass spectrum.

Revised from [5] using additional information from [65]. 


\section{0. charge-mediated fragmentation heterolytic fragmentation; inductive cleavage}

Fragmentation of an ion in which the cleavage of one of its bonds is induced by the charge site. The cleaved bond is adjacent to the apparent charge site, and its cleavage involves movement of the electron pair forming that bond to the original charge site and thus migration of the charge to the released fragment.

See also charge remote fragmentation $(C R F)$.

From [66].

\section{1. charge number, $z$}

Absolute value of charge of an ion divided by the value of the elementary charge $(e)$ rounded to the nearest integer.

See also $\mathrm{m} / \mathrm{z}$.

Revised from [5,6].

\section{2. charge permutation reaction}

Reaction of an ion with a neutral with a resulting change in the magnitude or sign of the charge on the reactant ion.

See also charge stripping reaction.

From $[5,6]$.

\section{3. charge remote fragmentation $(\mathrm{CRF})$}

Fragmentation of an ion in which the cleaved bond is isolated from the apparent charge site by a distance of several bond lengths.

See also charge-mediated fragmentation.

From $[67,68]$.

\section{4. charge site derivatization}

Chemical derivatization of molecules to introduce a fixed charge site, thus improving ionization efficiency by electrospray ionization or matrix-assisted laser desorption/ionization and directing subsequent ion fragmentation in a manner that facilitates structure identification.

Note: Examples are quaternary ammonium or phosphonium derivatization. From [69].

\section{5. charge stripping reaction (CSR)}

Reaction of an ion with a neutral or collision partner ion in which a free electron is stripped from the ion, resulting in the charge on the product ion being more positive than that of the reactant ion.

Note: A particular example of an ionizing collision.

See also charge permutation reaction, ionizing collision.

Revised from [5,6] using additional information from [70]. 
76. charge-transfer reaction (in mass spectrometry) charge exchange reaction

Reaction of an ion with a neutral or collision partner ion in which some or all of the charge on the reactant ion is transferred to the neutral or collision partner ion. Revised from [5,6] using additional information from [71].

\section{7. chemical ionization (CI)}

Formation of a new ion in the gas phase by the reaction of a neutral with an ion. The process may involve transfer of an electron, a proton, or other charged species between the reactants.

Note 1: When a positive ion results from chemical ionization, the term may be used without qualification. When a negative ion results, the term negative ion chemical ionization should be used.

Note 2: This term is not synonymous with chemi-ionization.

See also atmospheric pressure chemical ionization.

From $[5,6]$.

\section{8. chemi-ionization}

Ionization of an atom or molecule by interaction with another internally excited atom or molecule.

Note 1: This term is not synonymous with chemical ionization.

Note 2: Chemi-ionization differs from Penning ionization in that chemical change is not excluded.

Revised from [5,6] using additional information from [72].

\section{9. c-ion}

Fragment ion containing the peptide N-terminus formed upon dissociation of a peptide ion at the peptide backbone $\mathrm{N}-\mathrm{C}$ bond.

From [28].

\section{0. cluster ion}

Ion formed by the combination via noncovalent forces of two or more atoms or molecules of one or more chemical species with an ion.

Note: For example, $\left[\left(\mathrm{H}_{2} \mathrm{O}\right)_{n} \mathrm{H}\right]^{+},\left[\left(\mathrm{H}_{2} \mathrm{O}\right)_{n}\left(\mathrm{CH}_{3} \mathrm{OH}\right)_{\mathrm{m}} \mathrm{H}\right]^{+},\left[(\mathrm{NaCl})_{n} \mathrm{Na}\right]^{+}, \mathrm{Au}_{10}{ }^{-}$, or $[\mathrm{M}+\mathrm{Na}+$ Revised from $[5,6]$. $\left.\mathrm{CH}_{3} \mathrm{OH}\right]^{+}$where $\mathrm{M}$ represents a molecule.

\section{1. coaxial reflectron}

Reflectron that is arranged such that its main axis is coaxial with the ion source and first stage of the time-of-flight analyzer.

From [73]. 


\section{2. collector slit}

Slit installed in a magnetic sector mass spectrometer to allow only those $m / z$ separated ions with a specific $m / z$ into the detector system.

From $[74,75]$.

\section{3. collisional activation $(\mathrm{CA})$}

See collisional excitation.

\section{4. collisional excitation collisional activation}

Interaction of an ion with another gas-phase species in which part of the relative translational energy of the collision partners is converted into internal energy.

From $[5,6]$.

\section{5. collisional focusing}

Effect where collisions with a buffer gas in a radio-frequency ion trap or transmission quadrupole causes ions to lose translational energy and concentrate at the center of the trap or axis of the linear quadrupole. The effect increases with gas pressure up to a point where ion scattering becomes the dominant process.

From [76].

\section{6. collisionally activated dissociation (CAD)}

See collision-induced dissociation (CID).

\section{7. collision cell}

Chamber in the ion path between $\mathrm{m} / \mathrm{z}$ separation elements, or between ion source acceleration region and the first analyzer, in tandem mass spectrometry in space configurations.

See also collision reaction cell.

From [77].

\section{8. collision gas}

Deprecated: target gas.

Inert gas used for collisional excitation or a reactive gas used for ion/molecule reactions.

See also buffer gas.

From [78].

\section{9. collision-induced dissociation (CID) collisionally activated dissociation}

Dissociation of an ion after collisional excitation.

Note: The terms collision-induced dissociation (CID) and collisionally activated dissociation

From $[5,6]$. $(C A D)$ can be used interchangeably. 


\section{0. collision quadrupole}

Transmission quadrupole to which an oscillating radio frequency potential is applied so as to focus a beam of ions through a collision gas or buffer gas with no $\mathrm{m} / \mathrm{z}$ separation other than low $\mathrm{m} / \mathrm{z}$ cut-off.

Note: Higher-order multipoles can also be used in this manner.

See also RF-only quadrupole.

From $[79,80]$.

\section{1. collision reaction cell (CRC)}

Collision cell for removal of interfering ions by ion/neutral reactions in inductively coupled plasmamass spectrometry.

From [81].

\section{2. concentric nebulizer}

Pneumatic nebulizer in which the liquid flows through a central capillary and dispersion gas flows through a surrounding outer tube.

From [82].

\section{3. cone voltage dissociation}

This term is deprecated.

See in-source collision-induced dissociation.

\section{4. consecutive reaction monitoring (CRM)}

Multiple-stage mass spectrometry experiment with three or more stages of $\mathrm{m} / \mathrm{z}$ separation in which products of sequential fragmentation or bimolecular reactions are selected for detection.

See also multiple reaction monitoring.

From [83].

\section{5. constant neutral mass gain spectrum \\ fixed neutral gain spectrum \\ fixed neutral mass gain spectrum}

Spectrum formed of all precursor ions that have undergone a selected $\mathrm{m} / \mathrm{z}$ increase corresponding to the mass of a particular neutral species divided by the charge number of the precursor. The ions detected are the product ions produced by gain of a preselected neutral species mass following ion/molecule reactions of the precursor ions with a reactant gas in a collision cell.

See also constant neutral mass loss spectrum.

From $[5,6]$.

\section{6. constant neutral mass loss spectrum \\ fixed neutral loss spectrum \\ fixed neutral mass loss spectrum}

Spectrum of all precursor ions that have undergone a selected $\mathrm{m} / \mathrm{z}$ decrement corresponding to the mass of a particular neutral divided by the charge number of the precursor. The ions detected are the product 
ions produced by loss of a preselected neutral mass following collision-induced dissociation of the precursor ions in a collision cell.

See also constant neutral mass gain spectrum.

From $[5,6]$.

\section{7. continuous dynode particle multiplier channel electron multiplier}

Ion-to-electron detector in which the ion strikes the inner surface of a continuous tube device and induces the production of secondary electrons that, in turn, impinge on the inner surfaces of the device to produce more secondary electrons. This avalanche effect produces an increase in the final measured current pulse.

See also discrete dynode particle multiplier.

From [84].

\section{8. continuous-flow fast atom bombardment (CF-FAB)}

Variant of fast atom bombardment in which the mixture of analyte and liquid matrix is continuously supplied to the sample probe tip.

From $[85,86]$.

\section{9. continuous-flow matrix-assisted laser desorption/ionization (CF-MALDI)}

Variant of matrix-assisted laser desorption/ionization in which the analyte is dissolved in a liquid matrix that is continuously supplied to the sample probe tip.

From [87].

\section{0. conversion dynode}

Surface that is held at high potential so that ions striking the surface produce secondary charged particles that are subsequently detected.

Note: For positive ions, the potential is negative and the secondary particles are electrons and negative ions. For negative ions, the potential is positive and the secondary particles are positive ions.

From $[88-90]$.

\section{1. corona discharge}

Electric discharge generated in a gas, usually at atmospheric pressure, surrounding a conductor carrying a sufficiently high potential that the electric field around it ionizes the gas so as to form a plasma in the immediate vicinity of the conductor without causing complete electric breakdown or arcing.

Note: A corona discharge can also be sustained in a liquid.

See also glow discharge ionization.

From [91].

\section{2. corona discharge ionization}

Formation of ions in a corona discharge.

From [92].

(C) 2013, IUPAC

Pure Appl. Chem., Vol. 85, No. 7, pp. 1515-1609, 2013 


\section{3. counter-current gas}

Stream of inert gas, often heated, that is used in an electrospray or other spray ionization source to aid in the evaporation of solvent from the sprayed particles. The flow of the gas is in the direction opposite to that of the spray.

Note: $\quad$ Curtain gas is a proprietary term used to indicate a flow of counter-current gas emanating from between two closely spaced plates each with a central orifice along the axis of a spray ionization source. The term should only be used to describe the commercial device.

See also drying gas.

From $[82,93,94]$.

\section{4. counter electrode}

Counter electrode in an electrospray ionization ion source is one of two electrodes between which the high electric potential is applied, the other electrode being the electrospray needle.

From [95].

\section{5. crossed electric and magnetic fields}

Electric and magnetic fields with the electric field direction at right angles to the magnetic field direction.

See also Wien filter.

From $[5,6]$.

\section{6. cross-flow nebulizer}

Pneumatic nebulizer in which the dispersion gas flows at an angle, usually $90^{\circ}$, with respect to the column of liquid emerging from a capillary.

From $[95,96]$.

\section{7. curved field reflectron}

Reflectron designed to provide a decelerating field strength that varies with depth of ion penetration and thus with ion translational energy, permitting energy focusing of ions with a range of translational energies such as those formed as product ions from post-source decay of metastable ions.

From [97].

\section{8. cycloidal mass spectrometer}

This term is deprecated.

See prolate trochoidal mass spectrometer.

\section{9. cyclotron motion}

Circular motion of a charged particle moving perpendicularly to the direction of a magnetic field that results from the Lorentz force.

From [15]. 
110. dalton, Da

See unified atomic mass unit.

\section{Daly detector}

Detector consisting of an off-axis conversion dynode, scintillator, and photomultiplier. The dynode at high potential emits secondary electrons when ions impinge on the surface. The secondary electrons are accelerated onto the scintillator that produces light that is then detected by the photomultiplier detector, which can be outside the vacuum system.

See also ion-to-photon detector.

From [98].

\section{2. data-dependent acquisition}

Mode of data collection in tandem mass spectrometry in which a fixed number of precursor ions whose $\mathrm{m} / \mathrm{z}$ values were recorded in a survey scan are selected using predetermined rules and are subjected to a second stage of mass selection in an $M S / M S$ analysis.

From [99].

\section{3. daughter ion}

This term is deprecated.

See product ion.

\section{4. daughter ion analysis}

This term is deprecated.

See product ion analysis.

\section{5. deconvoluted mass spectrum}

Mass spectrum processed with an algorithm designed to extract a desired signal or signals from raw experimental data in which the desired signals have been complicated (convolved) by some interferences or in some other way.

Note: $\quad$ Examples of deconvoluted mass spectra include mass spectra corrected for instrumental mass bias, or extraction of spectra for pure components from the spectrum of a mixture, or a mass (not $\mathrm{m} / \mathrm{z}$ ) spectrum of a biopolymer from an electrospray ionization specFrom $[100,101]$. trum containing peaks corresponding to several charge states.

\section{6. delayed extraction (DE)}

Application of the accelerating voltage pulse after a time delay in desorption ionization from a surface.

Note: The extraction delay can produce energy focusing in a time-of-flight mass spectrometer. See also time-lag focusing.

From $[102,103]$. 


\section{7. delta notation}

See relative isotope-ratio difference.

\section{8. deprotonated molecule}

Deprecated: pseudo-molecular ion, quasi-molecular ion.

Ion formed by the removal of a proton from a molecule $\mathrm{M}$ to produce an anion represented as $[\mathrm{M}-\mathrm{H}]^{-}$.

See also protonated molecule.

From [104].

\section{9. desorption chemical ionization (DCI)}

Formation of gas-phase ions by chemical ionization of gaseous molecules produced by the rapid heating and desorption of a solid or low vapour pressure liquid.

From $[105,106]$.

\section{0. desorption electrospray ionization (DESI)}

Formation of gas-phase ions from a solid or liquid sample at atmospheric pressure through the interaction of electrosprayed droplets with the sample surface.

See also ambient ionization.

From [107].

\section{1. desorption ionization}

Formation of gas phase ions from a solid or liquid sample surface upon activation by heat, strong electric field, particles, droplets, or photon bombardment.

From [108].

\section{2. desorption ionization on silicon (DIOS)}

Soft ionization alternative to matrix-assisted desorption/ionization involving laser desorption ionization of a sample deposited on a porous silicon surface.

See also matrix-assisted laser desorption/ionization.

From [109].

\section{3. detection limit}

See relative detection limit.

\section{4. diagnostic ion}

Product ion whose formation reveals structural or compositional information about its precursor ion.

Note: $\quad$ For example, the phenyl cation in an electron ionization mass spectrum is a diagnostic ion for benzene and derivatives.

From [110]. 


\section{5. dielectric barrier discharge ionization}

Atmospheric pressure chemical ionization in which the low-temperature plasma is not generated by a corona discharge but by a device consisting of two electrodes with at least one dielectric layer that separates the electrode from the plasma.

From [111].

\section{6. dimeric ion}

Ion formed by ionization of a dimer or by the association of an ion such as $[\mathrm{M}]^{+\bullet},[\mathrm{M}+\mathrm{H}]^{+}$, or $[\mathrm{M}-\mathrm{H}]^{-}$ with its neutral counterpart M.

See also adduct ion.

From $[5,6]$.

\section{7. d-ion}

High-energy fragment ion of a protonated peptide, formally equivalent to a fragment ion formed by homolytic cleavage of a peptide $\mathrm{C}-\mathrm{N}$ bond together with the part of the $\mathrm{C}$-terminal side-chain starting at the $\gamma$-carbon (if any).

From [112].

\section{8. direct analysis of daughter ions (DADI)}

This term is deprecated.

See mass-analyzed ion kinetic energy spectrometry (MIKES).

\section{9. direct exposure probe (DEP)}

Device based on a modification of a direct insertion probe for exposing an involatile sample directly to the electron beam (in an electron ionization source) or to a chemical ionization plasma as in desorption chemical ionization.

Note: The sample can be deposited on an inert surface or on a heater filament for rapid heating.

From [105,113].

\section{0. direct infusion}

Method of liquid sample introduction in which the sample is continuously flowed into a mass spectrometer ion source.

From [114].

\section{1. direct insertion probe (DIP)}

Device for introducing a single sample of a solid or liquid, usually contained in a quartz or other nonreactive sample holder, into a mass spectrometer ion source.

See also direct exposure probe.

From [115]. 


\section{2. direct liquid introduction (DLI)}

Direct delivery of a liquid sample at a low solvent flow rate into the chemical ionization ion source of a mass spectrometer. The solvent vapour can act as the reagent gas.

From [116].

\section{3. discrete dynode particle multiplier}

Ion-to-electron detector in which the ion strikes the surface of an electrode and induces the production of secondary electrons. In turn, these impinge in sequence on dynodes at a more positive electric potential to produce more secondary electrons. This avalanche effect produces an amplification of several orders of magnitude in the final measured current pulse.

See also continuous dynode particle multiplier.

From [117].

\section{4. dissociative electron capture}

Ionization mechanism in which capture of a low-energy electron by a molecule leads directly to a dissociative state of the nascent anion, an example of dissociative ionization.

From [118].

\section{5. dissociative ionization}

Reaction of a gas-phase molecule that results in its decomposition to form products, one of which is an ion.

See also associative ionization.

From $[5,6]$.

\section{6. distonic ion}

Radical ion whose charge and radical sites are neither at the same atom nor in conjugation with each other.

Note 1: For example, ${ }^{\bullet} \mathrm{CH}_{2}-\mathrm{OH}_{2}{ }^{+}$is a distonic ion, whereas the molecular ion of methanol, $\mathrm{CH}_{3} \mathrm{OH}^{+\bullet}$ is a conventional radical cation.

Note 2: In a valence bond description, $\alpha$-distonic ions have the charge and radical sites on adjacent atoms, $\beta$-distonic ions are separated by one atom, $\gamma$-distonic ions by two atoms, and so forth.

From $[119,120]$.

\section{7. double-focusing mass spectrometer}

Mass spectrometer that incorporates a magnetic sector and an electric sector connected in series in such a way that ions with the same $\mathrm{m} / \mathrm{z}$ but with distributions in both the direction and the translational energy of their motion are brought to a focus.

From $[5,6]$. 


\section{8. drift tube}

Cylindrical chamber containing a buffer gas. Ions introduced at one end are caused to move to the other end by either a flow of the buffer gas or by a uniform longitudinal electric field.

From [121].

\section{9. drying gas}

Inert gas that is used to promote the removal of solvent from aerosol particles in spray ionization.

See also counter-current gas.

From [122].

\section{0. dynamic exclusion}

Software method used to minimize repeat selections of identical precursor ions for collision-induced dissociation in replicate chromatography-tandem mass spectrometry analyses of complex mixtures.

See also data-dependent acquisition.

From [123].

\section{1. dynamic field mass spectrometer}

Mass spectrometer in which $\mathrm{m} / \mathrm{z}$ separation is achieved using one or more electric fields that vary with time.

From $[5,6]$.

\section{2. dynamic secondary ion mass spectrometry (DSIMS)}

Secondary ion mass spectrometry analysis with primary ion current density sufficiently high for use in analysis of sample surface layers in the depth direction.

From [124].

\section{3. einzel lens}

Three-element charged particle lens in which the first and third elements are maintained at the same electric potential. Such a lens produces focusing without changing the translational energy of the particle.

From [125].

\section{4. elastic scattering}

Interaction between two particles (atom, molecule, or ion) wherein there is an exchange of translational energy between the collision partners but no conversion of translational energy into internal energy. Both the magnitudes and directions of the particle speeds can change.

See also inelastic scattering.

Revised from $[5,6]$.

\section{5. electric sector}

See electrostatic energy analyzer. 


\section{6. electrohydrodynamic ionization}

Ionization process that produces charged droplets and gas-phase ions from a liquid under vacuum with the application of an electric field.

Note: The process is formally equivalent to sub-ambient pressure electrospray ionization. From [126-128].

147. electron affinity, $E_{\mathrm{EA}}$

Minimum energy required for a species $\mathrm{M}$ to be formed in the process $\mathrm{M}^{-\bullet} \rightarrow \mathrm{M}+e^{-}$where $\mathrm{M}^{-\bullet}$ and $\mathrm{M}$ are in their ground rotational, vibrational, and electronic states and the electron has zero translational energy.

From $[5,6]$.

148. electron attachment ionization

This term is deprecated.

See electron capture ionization.

\section{9. electron capture dissociation (ECD)}

Process in which multiply protonated molecules interact with low-energy electrons followed by fragmentation. The capture of an electron by the even-electron cation $[\mathrm{M}+n \mathrm{H}]^{n+}$ liberates energy and reduces its charge state, thus producing the corresponding $[\mathrm{M}+n \mathrm{H}]^{(n-1)+\bullet}$ odd-electron ion, which readily fragments.

From [129].

150. electron capture ionization (ECI) electron capture negative ionization

Deprecated: electron attachment ionization.

Ionization of a gas-phase atom or molecule by attachment of a free electron to form molecular ions of the $\mathrm{M}^{\bullet}$ type.

From [130].

151. electron capture negative ionization (ECNI)

See electron capture ionization (ECI).

\section{2. electron energy}

Translational energy that electrons acquire when accelerated in an electric field.

From $[5,6]$.

\section{3. electron impact ionization}

This term is deprecated.

See electron ionization (EI). 


\section{4. electron ionization (EI)}

Deprecated: electron impact ionization.

Ionization that removes one or more electrons from an atom or molecule through interactions with electrons that are typically accelerated to energies between 10 and $150 \mathrm{eV}$.

From $[5,6]$.

\section{5. electron transfer dissociation}

Process in which multiply protonated molecules accept an electron from an anion with relatively low electron affinity. Capture of the electron leads to the liberation of energy and a reduction in charge of the ion with the production of the $[\mathrm{M}+n \mathrm{H}]^{(n-1)+\bullet}$ odd-electron ion, which is readily fragmented by collision-induced dissociation.

See also electron capture dissociation (ECD).

From [131].

\section{6. electronvolt, $\mathrm{eV}$}

Non-SI unit of energy (symbol eV) defined as the energy acquired by a particle containing charge equal to elementary charge accelerated through a potential difference of one volt.

Note: An electronvolt is equal to $1.602176565(35) \times 10^{-19} \mathrm{~J}$ where the digits in parentheses indicate the estimated uncertainty in the final two digits of the value.

Revised from [6] using additional information from [46].

\section{7. electrospray emitter}

See electrospray needle.

\section{8. electrospray ionization (ESI)}

Spray ionization process in which either cations or anions in solution are transferred to the gas phase via formation and desolvation at atmospheric pressure of a stream of highly charged droplets that result from applying a potential difference between the tip of the electrospray needle containing the solution and a counter electrode.

Note: When a pressurized gas is used to aid in the formation of a stable spray, the term pneumatically assisted electrospray ionization is used. The term ionspray is deprecated From $[132,133]$. unless describing the commercial product.

\section{9. electrospray needle electrospray emitter}

Narrow bore tube from which highly charged droplets are emitted in electrospray ionization. From [95,133]. 
160. electrostatic energy analyzer (ESA) electric sector

Device consisting of pairs of parallel conducting plates, concentric cylinders, or concentric spheres that separate charged particles according to their ratio of translational energy to charge by means of a potential difference applied between the pair.

From [134].

\section{1. even-electron ion}

Ion containing no unpaired electrons in its ground electronic state.

From $[5,6]$.

\section{2. even-electron rule}

Rule that states that even-electron ions tend to form even-electron fragment ions whereas odd-electron ions tend to dissociate to form either odd- or even-electron ions.

From [135].

\section{3. exact mass}

Calculated mass of an ion or molecule with specified isotopic composition.

See also accurate mass.

From [136].

\section{4. extracted ion chromatogram}

mass chromatogram reconstructed ion chromatogram

Chromatogram created by plotting the intensity of the signal observed at a chosen $\mathrm{m} / \mathrm{z}$ value or set of values in a series of mass spectra recorded as a function of retention time.

From $[137,138]$.

\section{5. extracted ion electropherogram extracted ion electrophorogram}

Electropherogram created by plotting the intensity of the signal observed at a chosen $\mathrm{m} / \mathrm{z}$ value or set of values in a series of mass spectra recorded as a function of electrophoresis time.

From $[139,140]$.

\section{6. extracted ion electrophorogram}

See extracted ion electropherogram.

\section{7. extracted ion profile}

Any plot of signal intensity observed at a chosen $\mathrm{m} / \mathrm{z}$ value or set of values in a series of mass spectra recorded as a function of time, including extracted ion chromatogram, extracted ion electropherogram, or from flow injection analysis or any other time-dependent sampling. 
See also total ion current profile.

From [141].

\section{Faraday cup}

Conducting cup or chamber that intercepts a charged particle beam and is electrically connected to a current measuring device.

From $[5,6]$.

\section{9. fast atom bombardment ionization (FAB)}

Ionization of molecules initiated by a beam of neutral atoms with translational energies in the $\mathrm{keV}$ range. Labile molecules susceptible to dissociation are dissolved in an involatile liquid matrix that absorbs the energy of the bombarding atoms to form a selvedge region above the liquid surface in which ionization of the molecules occurs.

Note: $\quad$ Positive ions (e.g., protonated or cationized molecules) or negative ions (e.g., deprotonated molecules) are formed depending on the polarity of ionization.

See also liquid secondary ionization.

Revised from [5,6] using additional information from [142].

\section{0. fast ion bombardment (FIB)}

Ionization of any species by the interaction of a focused beam of ions, with a translational energy of several thousand $\mathrm{eV}$, with a solid or liquid sample.

Note: For a liquid sample, fast ion bombardment is the same as liquid secondary ionization. From [143].

\section{1. field desorption (FD)}

Deprecated: field desorption ionization.

Formation of gas-phase ions from a material deposited on a solid surface (known as an "emitter") in the presence of an electric field.

Note: Because this process probably encompasses ionization by field ionization in conjugation with other mechanisms of ionization, the term field desorption ionization, despite its widespread use, is imprecise and therefore not recommended.

From $[5,6]$.

\section{2. field desorption ionization}

This term is deprecated.

See field ionization.

\section{3. field-free region (FFR)}

Section of a mass spectrometer in which there are no electric or magnetic fields. From [144]. 


\section{4. field ionization (FI)}

Ionization by the removal of electrons from any gas-phase species via the action of a high electric field. See also field desorption.

From $[5,6]$.

\section{5. first stability region}

Stability region of a Mathieu stability diagram closest to the origin.

Note: Ions within this region can traverse the full length of a transmission quadrupole or can From [145]. be trapped in a Paul ion trap.

\section{6. fission fragment ionization}

See plasma desorption ionization.

\section{7. fixed neutral gain spectrum}

See constant neutral mass gain spectrum.

\section{8. fixed neutral loss spectrum}

See constant neutral mass loss spectrum.

\section{9. fixed neutral mass gain spectrum}

See constant neutral mass gain spectrum.

\section{0. fixed neutral mass loss spectrum}

See constant neutral mass loss spectrum.

\section{1. fixed precursor ion scan}

Scan that determines, in a single experiment, the $\mathrm{m} / \mathrm{z}$ values of product ions that are produced by the reaction of a selected precursor ion with a user-specified $m / z$ value.

From $[5,6]$.

\section{2. fixed precursor ion spectrum}

Spectrum obtained when data are acquired that determine the $\mathrm{m} / \mathrm{z}$ values of product ions produced by the reaction of a selected precursor ion with a user-specified $\mathrm{m} / \mathrm{z}$ value.

From $[5,6]$.

\section{3. fixed product ion scan}

Scan that determines, in a single experiment, the $\mathrm{m} / \mathrm{z}$ values of precursor ions that react to produce a product ion with a user-selected $\mathrm{m} / \mathrm{z}$ value.

From $[5,6]$. 


\section{4. flow injection analysis mass spectrometry}

See flow injection mass spectrometry.

\section{5. flow injection mass spectrometry flow injection analysis mass spectrometry}

Method of flow injection analysis in which an aliquot of sample is injected, without chromatographic separation, into a liquid carrier stream for on-line mass spectrometry. From [146,147].

\section{6. focal plane detector}

Detector for $\mathrm{m} / \mathrm{z}$ spatially dispersed ion beams in which all ions simultaneously impinge on the detector plane to produce spatially defined signals.

From [148].

\section{7. forward geometry} normal geometry

Double-focusing mass spectrometer configuration in which the ion beam traverses the electric sector before the magnetic sector.

See also reverse geometry.

From [149].

\section{8. forward library search}

Procedure of comparing a mass spectrum of an unknown compound with a mass spectral library so that the unknown spectrum is compared in turn with the library spectra, considering only the $m / z$ peaks that have intensities greater than a specific threshold in the unknown.

Note: In a forward library search, a higher similarity index indicates the extent to which the library spectrum resembles the unknown spectrum.

See also reverse library search.

From [150].

\section{Fourier transform-ion cyclotron resonance-mass spectrometer (FT-ICR-MS)}

Mass spectrometer based on the principle of ion cyclotron resonance in which an ion in a magnetic field moves in a circular orbit at the cyclotron frequency that is characteristic of its $\mathrm{m} / \mathrm{z}$ value. Ions are coherently excited to a larger radius orbit using a pulse of radio frequency electric field, and their image charge is detected on receiver plates as a time domain signal. Fourier transformation of the time domain signal results in a frequency domain signal that is converted to a mass spectrum based on the inverse relationship between cyclotron frequency and $\mathrm{m} / \mathrm{z}$.

See also ion cyclotron resonance mass spectrometer. From $[5,6]$. 


\section{Fourier transform mass spectrometry}

Mass spectrometry technique in which $\mathrm{m} / \mathrm{z}$ values are represented by frequencies of ion motion and mass spectra are generated by Fourier transform mathematical operations from time domain transients produced by image current detection.

Note: $\quad$ Fourier transform mass spectrometry is typically carried out using Fourier transformion cyclotron resonance-mass spectrometers or Kingdon traps.

From [151].

191. fragmentation reaction (in mass spectrometry)

Reaction of an ion that results in two or more fragments of which at least one is an ion.

Revised from [6].

\section{2. fragment ion}

Product ion that results from the dissociation of a precursor ion.

Revised from [5,6] using additional information from [152,153].

\section{3. fringe field}

Electric or magnetic field that extends from the edge of an electric sector, magnetic sector, lens, or other ion optics element.

From [154].

194. gas chromatography-mass spectrometry (GC-MS) gas chromatography/mass spectrometry (GC/MS)

Technique by which a mixture is separated into individual components by gas chromatography, followed by detection with a mass spectrometer.

Note 1: Either a hyphen or slash can be used to indicate combined instruments.

Note 2: The mass spectrum is typically obtained on a time scale that allows resolution of the component in a single chromatographic peak.

See also liquid chromatography-mass spectrometry $(L C-M S)$, supercritical fluid chromatography-mass spectrometry (SFC-MS), thin layer chromatography-mass spectrometry (TLC-MS).

From $[18,155]$.

\section{5. glow discharge ionization}

Formation of ions from gaseous atoms or molecules or from the vapor of a solid sample at the cathode by applying a potential difference between two electrodes immersed in a low-pressure inert gas environment.

From $[156,157]$.

\section{6. granddaughter ion}

This term is deprecated.

See $n^{\text {th }}$-generation product ion. 


\section{7. gridless reflectron}

Reflectron design in which the decelerating/accelerating field is produced without the use of wire-mesh grids.

Note: This design improves ion transmission but at the cost of a less homogeneous field and thus reduced resolution.

From [158].

\section{8. hard ionization}

Formation of gas-phase ions accompanied by extensive fragmentation.

See also soft ionization.

From [24].

199. high-energy collision-induced dissociation

Collision-induced dissociation process wherein the projectile ion has laboratory-frame translational energy greater than a given value, often taken to be $1 \mathrm{keV}$.

See also low-energy collision-induced dissociation.

From $[159,160]$.

200. high-field asymmetric waveform ion mobility spectrometry (FAIMS) RF-DC ion mobility spectrometry

Separation of ions at atmospheric pressure between two electrodes due to simultaneous application of a high potential asymmetric waveform, whereby ions migrate towards one of the two electrodes depending on the ratio of the high- to low-field mobility of the ion. A variable compensating DC potential is used to selectively transmit ions of interest.

From $[161,162]$.

\section{1. hybrid mass spectrometer}

Mass spectrometer that combines $\mathrm{m} / \mathrm{z}$ analyzers of different types to perform tandem mass spectrometry or higher-order $\mathrm{MS}^{n}$ experiment.

From [5].

\section{2. hydride ion}

See hydron.

\section{3. hydrogen/deuterium exchange (HDX)}

Exchange of hydrogen atoms with deuterium atoms in a chemical species in solution prior to introduction into a mass spectrometer, or by ion/molecule reaction with a neutral gas inside a mass spectrometer.

From $[163,164]$. 


\section{4. hydrogen shifts}

Sigmatropic reactions of ions in which the $\sigma$-bonds broken and formed involve hydrogen atoms, and are subject to the Woodward-Hofmann rules resulting from orbital symmetry considerations.

From [165].

\section{5. hydron}

hydride ion

General name for the cation $\mathrm{H}^{+}$.

Note 1: The species $\mathrm{H}^{-}$is the hydride anion, and $\mathrm{H}$ is the hydro group.

Note 2: These are general names to be used without regard to the nuclear mass of the hydrogen entity, either for hydrogen in its natural isotopic abundance or where it is not desired to distinguish among the isotopes.

From [6].

\section{6. hyphenated mass spectrometry technique}

Analytical technique in which mass spectrometry is interfaced with a pretreatment step, most often chromatographic separation but many other combinations are possible.

Note: Despite the use of the term "hyphenated" in the name of the general category, a slash From $[166,167]$. separator as in gas chromatography/mass spectrometry may also be used.

\section{7. image current detection}

Method of ion detection by measuring the current that results from the image charge induced by a coherent motion of ions passing near a conductor.

Note: In Fourier transform mass spectrometers or Kingdon traps, the coherent orbital motion of ions between a pair of electrodes induces a radio frequency image charge in the electrodes and an alternating image current in the detection circuit connecting to the electrodes.

From $[168,169]$.

\section{8. imaging mass spectrometry}

Procedure used to form chemically selective images of an object based on the mass spectrometric detection of ions desorbed from its surface.

From [170].

\section{9. iminium ion}

\section{alkylideneaminylium ion}

Deprecated: imonium ion, immonium ion.

Cation with the structure $\mathrm{R}_{2} \mathrm{C}=\mathrm{N}^{+} \mathrm{R}_{2}$.

Note: The terms imonium ion and immonium ion are irregularly formed and should not be used.

From [6]. 


\section{0. imonium ion}

This term is deprecated.

See iminium ion.

\section{1. immonium ion}

This term is deprecated.

See iminium ion.

\section{2. impact parameter}

Distance of closest approach of two particles if they had continued in their original directions of motion at their original speeds without the influence of intermolecular forces.

From $[5,6]$.

\section{3. iminylium ion} alkylideneaminylium ion

Cation having the structure $\mathrm{R}^{1} \mathrm{R}^{2} \mathrm{C}=\mathrm{N}^{+}$.

Note 1: A contraction of alkaniminylium ion.

Note 2: A subclass of nitrenium ions.

From [6].

\section{4. inductively coupled plasma (ICP)}

Gas discharge ion source in which the energy is supplied to the plasma by electromagnetic induction. From [171].

\section{5. inductively coupled plasma-mass spectrometry (ICP-MS)}

Mass spectrometry technique based on coupling a mass spectrometer with an inductively coupled plasma as an ion source that both atomizes samples into their constituent atoms and ionizes them to form atomic cations.

Note: The technique is highly sensitive for a range of metals and several non-metals, and proFrom $[171,172]$. vides information on isotopic distributions.

\section{6. inelastic scattering}

Interaction between two particles (atom, molecule, or ion) wherein there is both an exchange of translational energy between the collision partners and a conversion of translational energy into internal energy. Both the magnitudes and directions of the particle speeds can change.

See also elastic scattering.

Revised from [5,6]. 


\section{7. infrared multiphoton dissociation (IRMPD)}

Dissociation of a reactant ion as a result of the absorption of multiple infrared photons.

See also Blackbody infrared radiative dissociation (BIRD).

From [173-175].

\section{8. in-source collisionally activated dissociation}

See in-source collision-induced dissociation.

219. in-source collision-induced dissociation

in-source collisionally activated dissociation

Deprecated: capillary exit fragmentation, cone voltage dissociation.

Dissociation of an ion as a result of collisional excitation during ion transfer from an atmospheric pressure ion source to the vacuum chamber of the mass spectrometer.

Note 1: This process is similar to ion desolvation but uses higher collision energy.

Note 2: Terms such as cone voltage dissociation and capillary exit fragmentation are designFrom [176]. specific versions of this term, and are deprecated.

220. intensity relative to base peak (in mass spectrometry)

relative intensity (in mass spectrometry)

Ratio of intensity of a resolved peak to the intensity of the resolved peak that has the greatest intensity (base peak). This ratio is generally measured as the normalized ratio of the heights of the respective peaks in the mass spectrum, with the height of the base peak taken as 100 .

Note: Intensity is a measure of detector response and should not be used to describe the abundance of ions.

From $[5,6]$.

\section{1. interaction distance}

Farthest distance of approach of two particles at which it is discernable that they will not pass at the impact parameter.

From $[5,6]$.

\section{2. interference}

Systematic error in the measurement of a signal caused by the presence of contaminants in a sample. From $[5,6]$.

\section{3. internal fragment}

Fragment ion of a peptide ion precursor formed by a double backbone cleavage that contains neither the original $\mathrm{C}$ - or $\mathrm{N}$-terminal residues. Typically a combination of $\mathrm{b}$ - and y-type cleavages or occasionally an a- and y-type combination.

From [177]. 


\section{4. internal standard}

See surrogate internal standard.

\section{5. ion}

Atomic, molecular, or radical species with a non-zero net electric charge. From [6].

\section{6. ion collector}

Device for the capture of selected ions such as a Faraday cup collector or an electron multiplier. From [6].

\section{7. ion cyclotron resonance-mass spectrometer (ICR-MS)}

Mass spectrometer in which the ions to be detected, with a selected value of $m / z$, absorb energy through the effect of a high-frequency electric field and a constant magnetic field perpendicular to the electric field. Maximum energy is gained by the ions that satisfy the cyclotron resonance condition and as a result they are separated from ions of different $\mathrm{m} / \mathrm{z}$.

See also Fourier transform-ion cyclotron resonance-mass spectrometer (FT-ICR-MS).

From $[5,6]$.

228. ion desolvation (in mass spectrometry)

Removal of solvent molecules clustered around a gas-phase ion by means of heating and/or collisions with gas molecules.

From [176].

\section{9. ion energy loss spectrum}

Spectrum that shows the loss of translational energy of ions involved in ion/neutral reactions.

See also ion kinetic energy spectrometry (IKES), mass-analyzed ion kinetic energy spectrometry (MIKES).

From $[5,6]$.

\section{0. ion enhancement ionization enhancement}

Phenomenon in which the ionization efficiency of a compound is increased by the presence of another compound.

Note: $\quad$ The effect is most significant in electrospray ionization but is also observed in atmospheric pressure chemical ionization and to a lesser extent in other ionization methods.

See also ion suppression, matrix effects.

From [178].

\section{1. ion evaporation model}

Mechanism for production of gas-phase ions from highly charged fine liquid droplets as in electrospray ionization that involves direct ejection of the ions from the droplet surface.

(C) 2013, IUPAC

Pure Appl. Chem., Vol. 85, No. 7, pp. 1515-1609, 2013 
Note: This mechanism is thought to be dominant for smaller molecules.

See also charged residue model.

From $[179,180]$.

\section{2. ion funnel}

Series of stacked ring electrodes with progressively decreasing inner diameter to which a combined radio frequency and fixed potential is applied. The resulting field focuses ions travelling along the central axis.

From [181].

\section{3. ion gate}

Set of plates or grid of wires in an ion mobility spectrometer, time-of-flight mass spectrometer, or other mass spectrometer that is used to apply a pulsed electric field with the purpose of selectively deflecting charged particles.

See also mass gate.

From [182].

\section{4. ionic dissociation}

Dissociation of an ion into another ion of lower mass and one or more neutral or ions with a lower charge.

From $[5,6]$.

\section{5. ion/ion reaction}

Reaction between two ions, generally of opposite polarity.

Note: The hyphenated term ion-ion reaction is superseded. From [183].

\section{6. ionization efficiency}

Ratio of the number of ions generated to the number of molecules consumed in the ion source of a mass spectrometer.

Note 1: The method for estimating the number of molecules consumed must always be clearly stated.

Note 2: In some older literature the ionization efficiency is defined as the fraction of electrons or photons that ionize a fixed number of molecules. The ionization efficiency varies with both the energy and the number of the ionizing entities, and in such cases an ionization efficiency curve can provide useful thermochemical information.

Note 3: The ionization efficiency strictly refers to the ionization events in the ion source. The fraction of molecules that are ionized and are subsequently detected also depends on the detection sensitivity of the instrument used.

Revised from [5,6] using additional information from [26,184]. 


\section{7. ionization efficiency curve}

Ionization efficiency as a function of the energy of the electrons or photons used to produce ionization of a fixed number of molecules.

From $[5,6]$.

\section{8. ionization energy, $E_{i}$}

Deprecated: ionization potential.

Minimum energy required to remove an electron from a neutral atom or molecule in its ground state.

Note 1: Adiabatic ionization energy refers to the formation of the molecular ion in its ground vibrational state and vertical ionization energy applies to the transition to the molecular ion without change in geometry.

Note 2: This quantity was formerly called ionization potential.

Note 3: Second ionization energy is the energy required to remove the second electron from the From $[5,6]$. singly charged ion.

\section{9. ionization enhancement}

See ion enhancement.

\section{0. ionization potential}

This term is deprecated.

See ionization energy.

\section{1. ionization suppression}

See ion suppression.

242. ionizing collision (in mass spectrometry)

Reaction of an ion with a neutral in which one or more electrons are removed from either the ion or neutral to form free electrons.

Note 1: This term has come to be used to describe collisions of fast-moving ions with a neutral in which the neutral is ionized with no change in the number of charges carried by the ion.

Note 2: Care should be taken when this term is used to emphasize whether or not charge stripping of the ion has taken place.

See also charge stripping reaction.

From $[5,6]$.

\section{3. ion kinetic energy spectrum (IKES)}

Spectrum obtained when a beam of ions is separated according to the translational energy-to-charge ratios of the ions contained in it.

(C) 2013, IUPAC

Pure Appl. Chem., Vol. 85, No. 7, pp. 1515-1609, 2013 
Note 1: A radial electric field $(E)$ achieves separation of the various ionic species in this way.

Note 2: In this context, kinetic energy is interpreted as translational, rather than vibrational or rotational kinetic energy.

See also ion energy loss spectrum, mass-analyzed ion kinetic energy spectrometry (MIKES).

From $[5,6]$.

\section{4. ion mobility spectrometry (IMS)}

Separation of ions according to their velocity through a buffer gas under the influence of an electric field.

From [185,186].

See also high-field asymmetric waveform ion mobility spectrometry (FAIMS).

\section{5. ion/molecule reaction}

Ion/neutral reaction in which the neutral is a molecule.

Note: The hyphenated formulation of this term, ion-molecule reaction, is deprecated; the hyphen suggests a reaction of a species that is both an ion and a molecule and is not the intended meaning.

See also ion/neutral reaction.

From $[5,6]$.

\section{6. ion/neutral complex}

Weakly bound species formed from an ion and neutral.

Note 1: In the context of some unimolecular ion dissociations, these complexes are particular types of transition states or local energy minima that lie between precursor ions and product ions on the reaction coordinate.

Note 2: The hyphenated formulation of this term, ion-neutral complex, is deprecated; the hyphen suggests a reaction of a species that is both an ion and a molecule and is not the intended meaning.

See also adduct ion.

From [187].

\section{7. ion/neutral reaction}

Process wherein a ion interacts with a neutral reactant to produce either chemically different species or changes in the internal energy of one or both of the reactants.

See also ion/molecule reaction.

From $[5,6]$.

\section{8. ion/neutral exchange reaction}

Reaction of a gas-phase ion with a neutral to produce a different neutral and ion as the products.

See also ion/neutral reaction.

From $[5,6]$. 


\section{9. ion optics}

Devices designed to control the formation, focusing, and deflection of charged particle beams in a vacuum under the influence of electric and magnetic fields.

See also mass spectrometer focusing system.

From [188].

250. ion pair formation (in mass spectrometry)

Gas-phase reaction of a molecule to form both positive ion and negative ion fragments among the products.

From $[5,6]$.

\section{1. ion source}

Region in a mass spectrometer where gas-phase ions are produced.

Revised from [6] using additional information from [189].

\section{2. ion suppression} ionization suppression

Phenomenon in which the ionization efficiency of a species is lowered by the presence of a different species.

Note: $\quad$ The effect is most significant in electrospray ionization but is also observed in atmospheric pressure chemical ionization and to a lesser extent in other ionization methods.

See also ion enhancement, matrix effects.

From [178].

\section{3. ion-to-photon detector}

Detector in which ions strike a conversion dynode to produce electrons that in turn strike a phosphor and the resulting photons are detected by a photomultiplier.

See also Daly detector.

From [190].

\section{4. ion trap (IT)}

Device for spatially confining ions using electric and magnetic fields alone or in combination.

Revised from [5,6] using additional information from [15,191].

255. isobar (in mass spectrometry)

Atomic or molecular species with the same nominal mass but different exact masses.

Note 1: In the case of atoms, this is equivalent to different nuclides of equal mass number.

Note 2: Isobaric ions are isobars that all carry an electric charge.

Note 3: This term is used in meteorology to denote lines connecting points of equal atmospheric pressure.

Revised from [6].

(C) 2013, IUPAC

Pure Appl. Chem., Vol. 85, No. 7, pp. 1515-1609, 2013 


\section{6. isobaric ion}

See isobar (in mass spectrometry).

\section{7. isobaric tag for relative and absolute quantitation (iTRAQ)}

Chemical labeling reagents used for relative or absolute quantitation in proteomics, based on covalent labeling of the N-terminus and side-chain amines of peptides from protein digestions with tags of varying mass.

From $[192,193]$.

\section{8. isotope cluster}

Group of peaks representing ions of the same elemental composition, but different isotopic compositions.

See also isotope pattern.

From [24].

\section{9. isotope coded affinity tag (ICAT)}

Chemical labeling reagents used for relative quantitation in proteomics, consisting of a reactive group capable of labeling a defined amino acid side-chain, an isotopically coded linker, and a tag for the affinity isolation of labeled proteins and peptides.

From [194].

\section{0. isotope delta}

See relative isotope-ratio difference.

\section{1. isotope dilution mass spectrometry (IDMS)}

Quantitative mass spectrometry technique in which an isotopically enriched compound is used as a surrogate internal standard.

From [195].

\section{2. isotope-number ratio}

See isotope ratio.

\section{3. isotope pattern}

Set of peaks related to ions with the same chemical formula but containing different isotopes that has a particular pattern associated with the relative abundance of the isotopes.

See also isotope cluster.

From $[5,6]$. 
264. isotope ratio, $R$

isotope-number ratio

Ratio of the number of atoms of one isotope to the number of atoms of another isotope of the same chemical element in the same system.

Note: Usually the minor, less abundant isotope to the major, most abundant isotope, for example, ${ }^{13} \mathrm{C} /{ }^{12} \mathrm{C}$.

From $[196,197]$.

265. isotope ratio mass spectrometry (IRMS)

stable isotope mass spectrometry

Measurement and study of the relative abundances of the different isotopes of an element in a material using a mass spectrometer.

From [198].

\section{6. isotopically enriched ion}

Ion enriched in an isotope in which the abundance of the particular nuclide is increased above the abundance at which it occurs in a reference, usually a naturally occurring sample.

From $[5,6]$.

\section{7. isotopic ion}

Any ion containing one or more of the less abundant naturally occurring isotopes of the elements that make up its structure.

Note: $\quad$ For example, $\mathrm{CH}_{2} \mathrm{D}^{+}$.

From $[5,6]$.

\section{8. isotopic scrambling}

Complete random mixing of isotopes between specified positions in an ion or neutral to achieve an equilibrium distribution of isotopes.

Note: In mass spectrometry, this term usually refers to the random mixing of isotopes in isolated ions that were formed with a fixed isotopic composition.

See also partial isotopic scrambling.

From $[5,6]$.

\section{9. isotopolog ions}

Ions that differ only in the isotopic composition of one or more of the constituent atoms.

Note 1: For example, $\mathrm{CH}_{4}{ }^{+}$and $\mathrm{CH}_{3} \mathrm{D}^{+\bullet}$ or ${ }^{10} \mathrm{BF}_{3}{ }^{+\bullet}$ and ${ }^{11} \mathrm{BF}_{3}{ }^{+\bullet}$, or the ions forming an isotope cluster corresponding to the natural isotopic abundances of the constituent atoms.

Note 2: Isotopolog is an abbreviation of isotopic homolog.

From $[6,10]$. 


\section{0. isotopomeric ion}

Isomeric ions having the same numbers of each isotopic atom but differing in their positions within the ion.

Note 1: Examples are $\mathrm{D}_{2} \mathrm{C}=\mathrm{CH}_{2}{ }^{+\bullet}$ and $\mathrm{HDC}=\mathrm{CHD}^{+\bullet}$.

Note 2: Isotopomeric ions can be either configurational isomers in which two atomic isotopes exchange positions or isotopic stereoisomers.

Note 3: Isotopomer is an abbreviation of isotopic isomer. From $[6,10]$.

\section{1. jet separator}

Gas chromatograph-to-mass spectrometer interface where the gas chromatography effluent expands into a lower-pressure region and then through a downstream orifice. The low mass carrier gas molecules diffuse from the jet axis, leaving the sample stream enriched in the heavier analyte molecules.

See also momentum separator.

From $[199,200]$.

\section{2. kinetic energy release (KER)}

Translational energy of fragments resulting from dissociation of a metastable ion measured relative to the center-of-mass.

Note: In this context, kinetic energy is interpreted as translational, rather than vibrational or rotational, kinetic energy.

See also kinetic energy release distribution (KERD).

From [201].

\section{3. kinetic energy release distribution (KERD)}

Distribution of values of translational energies in the center-of-mass reference for an ensemble of metastable ions undergoing a specific dissociation reaction.

Note: In this context, kinetic energy is interpreted as translational, rather than vibrational or rotational, kinetic energy.

See also mass-analyzed ion kinetic energy spectrometry (MIKES).

From [201].

\section{4. kinetic method}

Method for the determination of ion thermodynamic quantities by a procedure in which the relative probabilities of competing ionic dissociations are measured via the relative abundances of the reaction products.

Note: The extended kinetic method takes the associated entropy changes into account. Revised from [6]. 


\section{5. kinetic shift}

Excess energy above the thermochemical threshold required to observe dissociation of a precursor ion within the timeframe of a particular mass spectrometry experiment.

Note: Kinetic shifts are important when thermochemical quantities are deduced from appearance energy measurements.

From [202].

\section{Kingdon trap}

Ion trapping device that consists of an outer barrel-like electrode and a coaxial inner spindle-like electrode that form an electrostatic field with a quadro-logarithmic potential distribution.

Note 1: The frequency of harmonic oscillations of the orbitally trapped ions along the axis of the electrostatic field is independent of the ion velocity and is inversely proportional to the square root of $\mathrm{m} / \mathrm{z}$ so that the trap can be operated as a mass analyzer using image current detection and Fourier transformation of the time domain signal.

Note 2: The trademarked term Orbitrap has been used to describe a Kingdon trap used as a mass spectrometer.

From [203].

\section{7. label-free quantitation}

Method for determining the concentration of proteins through a combination of protein digestion and liquid chromatography-mass spectrometry ( $L C-M S)$ without relying on stable isotope labeling. Protein quantification is achieved by measuring the signal intensity of peptide ions corresponding to a protein or by counting and comparing the number of fragment mass spectra identifying peptides of a given protein.

From [204].

\section{8. laser ablation}

Removal of material from a solid or liquid sample using energy delivered by a short laser pulse to form gas-phase species and particles.

See also laser desorption.

From [205].

\section{9. laser desorption (LD)}

Formation of gas-phase species from a solid or liquid sample surface upon activation using a short pulse of photons from a laser.

See also laser ablation.

From [206].

\section{0. laser desorption ionization (LDI)}

Formation of gas-phase ions by the interaction of a laser with a solid or liquid material.

See also matrix-assisted laser desorption/ionization.

From [207]. 


\section{1. laser ionization $(\mathrm{LI})$}

Formation of ions through the interaction of photons from a laser with solid or liquid materials or with gas-phase atoms or molecules.

See also laser desorption ionization (LDI), photoionization.

From $[5,6]$.

\section{2. laser microprobe mass spectrometry (LMMS)}

Laser desorption ionization mass spectrometry, particularly emphasizing spatially resolved composition information.

From [208].

\section{3. linear ion trap (LIT)}

Two-dimensional Paul ion trap in which ions are confined in the axial dimension by means of a static electric potential.

From [209].

\section{4. linked scan}

Scan in a tandem mass spectrometer with two or more $\mathrm{m} / \mathrm{z}$ analyzers or in a sector mass spectrometer that incorporates at least one magnetic sector and one electric sector. The analyzers and/or other electric fields are scanned simultaneously so as to preserve a predetermined relationship between scan parameters to produce a product ion spectrum, precursor ion spectrum, constant neutral loss spectrum, or constant neutral mass gain spectrum.

From $[5,6]$.

\section{5. liquid chromatography-mass spectrometry (LC-MS) liquid chromatography/mass spectrometry (LC/MS)}

Technique by which a mixture of analytes is separated into individual components by liquid chromatography (typically high-performance liquid chromatography), followed by detection with a mass spectrometer.

Note: $\quad$ Either a hyphen or slash can be used to indicate combined instruments. From $[210,211]$.

\section{6. liquid ion evaporation}

Spray ionization interface in which droplets are dispersed from a pneumatic nebulizer toward a high potential electrode that produces a static charge on the droplets leading to ion formation after solvent evaporation.

From [94].

\section{7. liquid junction interface}

Means of coupling capillary electrophoresis to a mass spectrometer in which a liquid reservoir surrounds the separation capillary and transfer capillary to the mass spectrometer and provides electric contact for the capillary electrophoresis.

From [212,213]. 


\section{8. liquid secondary ionization (LSI)}

Ionization of any species by the interaction of a focused beam of ions or atoms with a sample that is dissolved in a solvent matrix.

See also fast atom bombardment ionization $(F A B)$.

From [214].

\section{9. liquid sheath}

Flow of make-up liquid in a sheath flow interface.

From [215-217].

\section{0. lock mass}

Mass of an ion of known $\mathrm{m} / \mathrm{z}$ value, derived from a suitable standard compound introduced into an ion source together with the sample to be analyzed, which permits real-time recalibration by correction of $\mathrm{m} / \mathrm{z}$ shifts arising from instrumental drift.

From [218].

\section{1. low-energy collision-induced dissociation}

Collision-induced dissociation process wherein the precursor ion has laboratory-frame translational energy lower than a given value, often taken to be $1 \mathrm{keV}$.

Note: This process can be carried out under single collision conditions but typically requires multiple collisions and the collisional excitation is cumulative.

See also high-energy collision-induced dissociation.

From [219].

\section{2. lyonium ion}

Cation produced by addition of a hydron to a solvent molecule.

Note: For example, $\mathrm{CH}_{3} \mathrm{OH}_{2}{ }^{+}$and $\mathrm{CH}_{3} \mathrm{OHD}^{+}$are both lyonium ions of methanol. See also onium compounds.

From [6].

\section{3. magnetic deflection}

Deflection of an ion beam as a result of the motion of the ions in a magnetic field.

Note 1: An example is a magnetic sector.

Note 2: Generally, the direction of deflection of the ions is perpendicular to both the direction From $[5,6]$. of the magnetic field and to that of the ion beam.

\section{4. magnetic field scan}

Method of producing a mass spectrum from a mono-energetic ion beam by varying the strength of the magnetic field in a sector mass spectrometer. 
Note 1: The magnetic field scan is sometimes called B-scan, but this is a misnomer since the symbol $B$ denotes the magnetic flux density. The magnetic field strength $H$ in a vacuum is defined in SI units as $H=\left(1 / \mu_{0}\right) B-M$ where $\mu_{0}$ is the magnetic constant or permeability of the vacuum and $M$ the magnetization. $B$ is often wrongly referred to as "magnetic field strength."

Note 2: A sector magnetic field produces a momentum/charge spectrum of an ion beam, but if all ions have the same translational energy this is equivalent to a mass spectrum. The force $F$ on a particle with charge $q$ and velocity $v$ is $F=q(v \times B)$.

From $[5,6]$.

\section{5. magnetic sector}

Device that produces a magnetic field perpendicular to a charged particle beam that deflects the beam to an extent that is proportional to the ratio of particle momentum and charge.

Note: For a mono-energetic beam, the deflection is proportional to $\mathrm{m} / \mathrm{z}$. From [5].

\section{6. magnetron motion}

Slow rotation of the center of cyclotron motion around the central axis of a Penning ion trap that results from a drift of the cyclotron centre along the electric equipotential lines perpendicular to the magnetic field direction.

Note: The characteristic frequency of this motion is called the magnetron frequency. From $[220,221]$.

\section{7. make-up liquid}

Solvent that is mixed with the sample flow in a liquid introduction mass spectrometry interface in order to adjust the flow rate or solvent characteristics of the system, for example, to promote ionization efficiency.

From [217].

\section{8. mass analysis}

Process by which a mixture of ionic or neutral species is identified according to their $\mathrm{m} / \mathrm{z}$ values (ions), or their aggregate atomic masses (neutrals), and their relative abundances. The analysis may be qualitative and/or quantitative.

From $[5,6]$.

\section{9. mass-analyzed ion kinetic energy spectrometry (MIKES)}

Deprecated: direct analysis of daughter ions (DADI).

Procedure by which translational energy spectra are obtained from a sector mass spectrometer that incorporates at least one magnetic sector plus one electric sector in reverse geometry (a magnetic sector followed by an electric sector).

Note 1: In this context, kinetic energy is interpreted as translational rather than vibrational or rotational kinetic energy. 
Note 2: The accelerating potential, $V$, and the magnetic flux density, $B$, are set at fixed values to $\mathrm{m} / \mathrm{z}$ select the precursor ions, which are then allowed to dissociate or to react in a field-free region between the two sectors. The translational energy-to-charge ratios of the various product ions are analyzed by scanning the electric sector field $E$. The width of a product ion spectral peak is related to the kinetic energy release distribution for the

From [222]. dissociation process.

\section{0. mass calibration}

Means of determining $\mathrm{m} / \mathrm{z}$ values of ions from experimentally detected signals using a theoretical or empirical relational equation.

Note: Most commonly this is accomplished using a computer-based data system and a calibration file obtained from a mass spectrum of a compound that produces ions of known $\mathrm{m} / \mathrm{z}$ values.

From [223].

\section{1. mass chromatogram}

See extracted ion chromatogram.

302. mass defect (in mass spectrometry)

Deprecated: mass excess.

Difference between the nominal mass and the monoisotopic mass of an atom, molecule, or ion.

Note: Mass defect can be a positive or negative value dependent upon the elemental composition.

From [224].

\section{3. mass discrimination}

Differences in overall detection sensitivities for ions of different $m / z$ values in a mass spectrum, caused by variations in ionization efficiency, transmission efficiency through the interface between the ion source and the analyzer vacuum system, analyzer, and detector response.

From [225].

\section{4. mass excess}

This term is deprecated.

See mass defect.

\section{5. mass fragmentography}

This term is deprecated.

See selected ion monitoring.

\section{6. mass gate}

Ion gate used to transmit ions in a given $\mathrm{m} / \mathrm{z}$ range.

(C) 2013, IUPAC

Pure Appl. Chem., Vol. 85, No. 7, pp. 1515-1609, 2013 
See also ion gate.

From [226].

\section{7. mass limit}

Value of $\mathrm{m} / \mathrm{z}$ above or below which ions cannot be detected in a mass spectrometer. From [227].

\section{8. mass mapping}

See peptide mass fingerprinting.

\section{9. mass number, $A$}

Sum of the numbers of protons and neutrons in an atom, molecule, or ion.

Note: When expressed as a mass in daltons, mass number is equivalent to nominal mass. From $[5,6]$.

\section{0. mass peak}

peak (in mass spectrometry)

Localized region of relatively intense detector response in a mass spectrum when ions of a specified $\mathrm{m} / \mathrm{z}$ are detected. If resolving power is insufficient two or more components of similar $\mathrm{m} / z$ may contribute to one unresolved mass peak.

Note 1: Although mass peaks are often associated with particular ions, the terms peak and ion should not be used interchangeably.

Note 2: Care should be used to distinguish mass spectrum peaks from chromatographic peaks Revised from [5,6]. in $G C-M S$ and $L C-M S$.

\section{1. mass range}

Range of mass over which a mass spectrometer can detect ions or is operated to record a mass spectrum.

Note: When indicating a range of $m / z$ instead of mass, this should be specified explicitly. From [6].

\section{2. mass resolution}

See resolution (in mass spectrometry).

\section{3. mass resolving power}

See resolving power (in mass spectrometry). 


\section{4. mass selective axial ejection}

Use of mass selective instability to eject ions of selected $m / z$ values from an ion trap along the trap axis. From [228].

\section{5. mass selective instability (MSI)}

Unstable trajectories in an ion trap for ions within a particular range of $m / z$ values caused by an appropriate combination of oscillating electric fields.

See also mass selective axial ejection.

From [229].

\section{6. mass spectral library}

Collection of mass spectra of different compounds, usually represented as arrays of signal intensity vs. the $m / z$ values.

From [230].

\section{7. mass spectrograph}

Mass spectrometer in which the ions are directed onto a focal plane detector such as a photographic plate.

From $[5,6]$.

\section{8. mass spectrometer}

Instrument that measures the $\mathrm{m} / \mathrm{z}$ values and abundances of gas-phase ions.

From $[5,6]$.

\section{9. mass spectrometer focusing system (deflection system)}

This term is obsolete.

Assembly in a sector mass spectrometer permitting the separation of ions according to their ratios of mass-to-charge.

See also ion optics.

From [6].

\section{0. mass spectrometry}

Obsolete: mass spectroscopy

Study of matter through the formation of gas-phase ions that are characterized using mass spectrometers by their mass, charge, structure, and/or physico-chemical properties.

Note 1: The term is a misnomer because it is $m / z$ rather than mass that is the independent variable in a mass spectrum.

Note 2: Mass spectroscopy is an obsolete synonym for mass spectrometry that should not be used to avoid confusion with spectroscopies in which the measured quantity is the

From $[5,6]$. absorption or emission of electromagnetic radiation. 


\section{1. mass spectrometry/mass spectrometry (MS/MS)}

tandem mass spectrometry

Acquisition and study of the spectra of the product ions or precursor ions of $\mathrm{m} / \mathrm{z}$ selected ions, or of precursor ions of a selected neutral mass loss.

Note: $\quad M S / M S$ can be accomplished using instruments incorporating more than one analyzer (tandem mass spectrometry in space) or in trap instruments (tandem mass spectrometry in time).

From [5].

\section{2. mass spectroscopy}

This term is obsolete.

See mass spectrometry.

\section{3. mass spectrum}

Plot of the relative abundances of ions forming a beam or other collection as a function of their $\mathrm{m} / \mathrm{z}$ values.

Note: $\quad$ The term is a misnomer because it is $m / z$ rather than mass that is the independent variable in a mass spectrum.

From $[5,6]$.

\section{4. mass-to-charge ratio}

This term is deprecated. The term "ratio of mass to charge" should be used for the quantity expressed in $\mathrm{kg} \mathrm{C}^{-1}$ in SI units.

See $m / z$.

\section{Mathieu stability diagram stability diagram}

Graphical representation expressed in terms of dimensionless reduced coordinates that describes the stability or instability of charged particle motion in a transmission quadrupole mass spectrometer or Paul ion trap, based on an appropriate form of the Mathieu differential equation.

From [231].

\section{6. matrix-assisted laser desorption/ionization (MALDI)}

Formation of gas-phase ions from molecules present in a solid or liquid matrix that is irradiated with a laser. The matrix is a material that absorbs the laser energy and promotes ionization.

From [232].

\section{7. matrix effects}

In quantitative analysis, especially with atmospheric pressure ionization techniques, matrix components co-extracted and co-eluting with the analytes can alter the ionization efficiency resulting in poor ana- 
lytical accuracy, linearity, and reproducibility. These effects can be either ionization suppression or ionization enhancement.

From [233].

\section{Mattauch-Herzog geometry}

Arrangement for a double-focusing mass spectrograph in which a deflection of $\pi /(4 \sqrt{2})$ rad in a radial electrostatic energy analyzer is followed by a magnetic deflection of $\pi / 2 \mathrm{rad}$.

See also Nier-Johnson geometry.

From $[5,6]$.

\section{McLafferty rearrangement}

Rearrangement reaction involving transfer of a hydrogen atom via a six-member transition state to the formal radical/charge site from a carbon atom four atoms distant from the charge/radical site (the $\gamma$-carbon); subsequent rearrangement of electron density can lead to expulsion of an olefin molecule.

Note: Originally applied to ketone molecular ions where the charge/radical site is the carbonyl Revised from $[5,6]$. oxygen, but is now more widely applied.

\section{0. membrane inlet mass spectrometry}

See membrane introduction mass spectrometry (MIMS).

\section{1. membrane interface mass spectrometry}

See membrane introduction mass spectrometry (MIMS).

\section{2. membrane introduction mass spectrometry (MIMS) membrane inlet mass spectrometry membrane interface mass spectrometry}

Mass spectrometry measurement that uses a semi-permeable membrane separator that permits the passage of analytes directly from solutions or ambient air to the mass spectrometer ion source.

From [234].

\section{3. membrane separator}

$G C-M S$ interface with a polymer membrane that selectively passes analyte molecules in preference to the carrier gas thereby enriching the analyte in the gas stream.

From [200].

\section{4. metastable ion}

Ion formed with internal energy higher than the threshold for dissociation but with a lifetime great enough to allow it to exit the ion source and enter the mass analyzer region where it dissociates before detection.

From $[5,6]$. 


\section{5. microchannel plate (MCP) \\ channel electron multiplier array}

Thin plate that contains a closely spaced array of channels each of which acts as a continuous dynode particle multiplier. A fast charged or neutral particle, or a photon, that strikes the plate causes a cascade of secondary electrons that ultimately exits the opposite side of the plate.

See also continuous dynode particle multiplier.

From [235].

\section{6. microelectrospray}

Electrospray ionization at a low solvent flow rate, typically several microliters per minute or lower, where a pump is used to provide the sample flow.

See also nanoelectrospray.

From [236].

\section{7. molar mass, $M$}

Mass of an element or chemical compound divided by its amount of substance.

Note: Amount of substance has historically been referred to as number of moles.

From $[6,7]$.

\section{8. molecular anion}

See also molecular ion.

From $[5,6]$.

\section{9. molecular beam mass spectrometry}

Mass spectrometry measurement in which the sample is introduced into the ion source of the mass spectrometer as a collimated beam of molecules with a narrow velocity distribution.

From [237].

\section{0. molecular cation}

See also molecular ion.

From $[5,6]$.

\section{1. molecular effusion separator}

$G C-M S$ interface that subjects the GC effluent to effusive flow through a porous glass tube. The lighter carrier gas passes out of the tube, leaving the gas within the tube enriched in analyte.

From $[200,238]$.

\section{2. molecular ion}

Ion formed by the removal of one or more electrons from a molecule to form a positive ion or the addition of one or more electrons to a molecule to form a negative ion. 
Note: Pseudo-molecular ion and quasi-molecular ion are deprecated; molecular ion is reserved for the intact ionized molecule with no component added or removed other than electrons.

From $[5,6]$.

\section{3. momentum separator}

Mass spectrometer interface to inlets including chromatography and spray-based ion sources that enriches the sample by selecting the central part of the effluent or spray using an orifice or skimmer. Particles with a higher forward momentum diverge less from the spray axis for a given radial momentum.

See also jet separator.

From $[239,240]$.

\section{4. monodisperse aerosol generating interface for chromatography (MAGIC)}

Particle beam interface that incorporates a monodisperse aerosol generator.

From [96].

\section{5. monoisotopic mass}

Exact mass of an ion or molecule calculated using the mass of the most abundant isotope of each element.

See also accurate mass.

From [51].

\section{6. monoisotopic mass spectrum}

Mass spectrum containing only ions made up of the principal isotopes of atoms of the original molecules.

See also monoisotopic mass.

From $[5,6]$.

\section{7. moving belt interface}

Liquid sample mass spectrometer interface that uses two or more pulleys and a continuous loop of material. Samples are sprayed or deposited from solution onto the belt where they are transported into the vacuum system for vaporization or desorption and ionization.

From [241].

\section{MS/MS spectrum}

Mass spectrum obtained using tandem mass spectrometry.

From [242].

\section{9. $M^{n}$}

Symbol for multiple-stage mass spectrometry experiments designed to record product ion spectra where $n$ is the number of product ion stages ( $n^{\text {th }}$-generation product ions).

From [243].

(C) 2013, IUPAC

Pure Appl. Chem., Vol. 85, No. 7, pp. 1515-1609, 2013 


\section{0. multicollector mass spectrometer}

Mass spectrograph based on a double-focusing magnetic analyzer that spatially disperses ions of different $\mathrm{m} / \mathrm{z}$ values on to an array of Faraday cup detectors, generally used with inductively coupled plasma ion sources for isotopic composition measurements.

From [244].

\section{1. multidimensional protein identification technology (MudPIT)}

Variant of shotgun proteomics in which proteins are first digested and then separated by a combination of strong cation exchange and reversed-phase liquid chromatography followed by mass spectrometry detection.

From [245].

\section{2. multiphoton ionization (MPI)}

Photoionization of an atom or molecule in which two or more photons are absorbed. From $[5,6]$.

\section{3. multiple ion detection (MID)}

This term is deprecated.

See selected ion monitoring.

\section{4. multiple reaction monitoring (MRM)}

Application of selected reaction monitoring to multiple product ions from one or more precursor ions.

Note: This term should not be confused with consecutive reaction monitoring, which involves From [246]. the serial application of three or more stages of selected reaction monitoring.

\section{5. multiple-stage mass spectrometry}

Multiple stages of precursor ion $\mathrm{m} / \mathrm{z}$ selection followed by product ion detection for successive $n^{\text {th }}$-generation product ions.

See also $M S^{n}$.

From [243].

\section{6. $\mathrm{m} / \mathrm{z}$}

Deprecated: mass-to-charge ratio, thomson.

Abbreviation representing the dimensionless quantity formed by dividing the ratio of the mass of an ion to the unified atomic mass unit, by its charge number (regardless of sign). The abbreviation is written in italicized lowercase letters with no spaces.

Note 1: Mass-to-charge ratio is deprecated. Mass-to-charge ratio has been used occasionally for the horizontal axis in a plot of a mass spectrum, although the quantity measured is not the ion's mass divided by its electric charge (SI units $\mathrm{kg} \mathrm{C}^{-1}$ ). However, $m / z$ is recommended as an abbreviation to represent the dimensionless quantity that is used almost universally as the independent variable in a mass spectrum. 
Note 2: The abbreviation $\mathrm{m} / \mathrm{z}$ should not be used as a variable in a mathematical equation. Instead, the variables $m$ (in $\mathrm{kg}$ ) and $q$ (in $\mathrm{C}$ ) should be used to denote mass and charge.

Note 3: The thomson (Th) unit [13] is deprecated.

From $[5,6]$.

\section{7. nanoelectrospray}

Electrospray ionization at a very low solvent flow rate, typically hundreds of nanoliters per minute of sample solution or lower, often without the use of an external solvent delivery system.

Note: Nanospray should only be used to describe the commercial product.

See also microelectrospray.

From [247].

\section{8. natural isotopic abundance}

Of a specified isotope of an element, the isotopic abundance in the element as found in nature, either an average value on planet Earth or from a specified location.

From $[5,6]$.

359. needle voltage

See nozzle-skimmer voltage.

360. negative ion

Atomic or molecular species having a net negative electric charge; an anion.

From $[5,6]$.

\section{1. negative ion chemical ionization (NICI)}

Chemical ionization that results in the formation of negative ions.

From [5].

\section{2. neutralization-reionization mass spectrometry (NRMS)}

Procedure by which neutral species are formed from $\mathrm{m} / \mathrm{z}$ selected ions by charge transfer to a collision gas or by dissociation. The neutral species are separated from the remaining ions and ionized through collisions with a second gas.

From [248].

\section{3. neutral loss}

Loss of an uncharged species from an ion during dissociation.

From $[5,6]$. 


\section{4. neutral loss scan}

Scan used to generate a constant neutral loss spectrum. From [249].

\section{Nier-Johnson geometry}

Arrangement for a double-focusing mass spectrometer in which a deflection of $\pi / 2 \mathrm{rad}$ in a radial electrostatic energy analyzer is combined with a magnetic deflection of $\pi / 3 \mathrm{rad}$, thus providing a single point of focus for ions of the same $\mathrm{m} / \mathrm{z}$ but with spreads in both trajectory direction and translational energy.

See also Mattauch-Herzog geometry.

Revised from [5,6] using additional information from [250].

\section{6. nitrenium ion}

Reactive intermediate based on nitrogen with both an electron lone pair and a positive charge and with two substituents $\left(\mathrm{R}_{2} \mathrm{~N}^{+}\right)$.

Note: The alkylidene derivatives of $\left(\mathrm{H}_{2} \mathrm{~N}^{+}\right),\left(\mathrm{R}_{2} \mathrm{C}=\mathrm{N}^{+}\right)$, still belong to the class nitrenium ions From [6]. but are more precisely designated by the term iminylium ions.

\section{7. nitrogen rule}

Rule stating that a neutral organic molecule containing one or more atoms of the elements $\mathrm{C}, \mathrm{H}, \mathrm{O}, \mathrm{N}$, $\mathrm{S}, \mathrm{P}$, or any halogen has an odd nominal mass if it contains an odd number of nitrogen atoms. From [24].

\section{8. nominal mass}

Mass of a molecular ion or molecule calculated using the isotope mass of the most abundant constituent element isotope of each element rounded to the nearest integer value and multiplied by the number of atoms of each element.

See also monoisotopic mass.

From [51].

\section{9. normal geometry}

See forward geometry.

\section{0. nozzle-skimmer dissociation in-source collision-induced dissociation}

Collisionally activated dissociation that is caused by collisions in the region between the nozzle and skimmer of an atmospheric pressure ionization source such as electrospray or atmospheric pressure chemical ionization.

Note: The process can be induced by a higher than typical nozzle-skimmer voltage. From $[251,252]$. 


\section{1. nozzle-skimmer voltage needle voltage}

Electric potential difference applied between the electrospray needle and the skimmer, which is typically held at the same potential as the counter electrode.

From $[252,253]$.

\section{2. $n^{\text {th }}$-generation product ion}

Deprecated: granddaughter ion.

Serial product ions from dissociation or bimolecular reaction of selected precursor ions where $n$ refers to the number of stages of dissociation or bimolecular reaction in a series of consecutive reactions that involve successive product ions.

Note 1: Given the sequential fragmentation scheme:

$$
\mathrm{M}_{1}^{+} \rightarrow \mathrm{M}_{2}^{+} \rightarrow \mathrm{M}_{3}^{+} \rightarrow \mathrm{M}_{4}^{+} \rightarrow \mathrm{M}_{5}^{+}
$$

$\mathrm{M}_{4}{ }^{+}$is the precursor ion of $\mathrm{M}_{5}{ }^{+}$, a $1^{\text {st }}$-generation product ion of $\mathrm{M}_{3}{ }^{+}$, a $2^{\text {nd }}$-generation See also $n^{\text {th }}$-generation product ion spectrum.

product ion of $\mathrm{M}_{2}^{+}$, and a $3^{\text {rd }}$-generation product ion of $\mathrm{M}_{1}{ }^{+}$.

From $[8,152,153]$.

\section{3. $n^{\text {th }}$-generation product ion spectrum}

Mass spectrum recorded from any mass spectrometer in which the appropriate scan function can be set to record the appropriate generation product ion or ions of $\mathrm{m} / \mathrm{z}$ selected precursor ions.

See also $n^{\text {th }}$-generation product ion.

From $[152,153]$.

\section{4. odd-electron ion}

See radical ion.

From $[5,6]$.

\section{5. onium compounds}

Cations derived by addition of a $\mathrm{H}^{+}$to a mononuclear parent hydride of the nitrogen, chalcogen, and halogen families.

Note: Examples are ammonium $\left(\mathrm{NH}_{4}^{+}\right)$, oxonium $\left(\mathrm{H}_{3} \mathrm{O}^{+}\right)$, and fluoronium $\left(\mathrm{H}_{2} \mathrm{~F}^{+}\right)$ions.

See also lyonium ion.

From [6].

\section{6. orthogonal acceleration}

See orthogonal extraction.

\section{7. orthogonal electrospray}

Configuration for electrospray ionization in which the axis of the spray is at a right angle to the axis of the sampling cone.

From [254].

(C) 2013, IUPAC

Pure Appl. Chem., Vol. 85, No. 7, pp. 1515-1609, 2013 


\section{8. orthogonal extraction} orthogonal acceleration

Pulsed acceleration of ions perpendicular to their original direction of travel into a mass spectrometer, typically time-of-flight.

Note: $\quad$ Ions may be extracted from a directional ion source, drift tube, or $\mathrm{m} / \mathrm{z}$ separation stage. From [252].

\section{9. parent ion}

This term is deprecated.

See precursor ion.

\section{0. parent ion spectrum}

This term is deprecated.

See precursor ion spectrum.

\section{1. partial charge exchange reaction}

See partial charge transfer reaction.

\section{2. partial charge transfer reaction partial charge exchange reaction}

Reaction of an ion with a neutral species in which some but not all of the ion charge is transferred to the neutral.

From $[5,6]$.

\section{3. partial isotopic scrambling}

Partial random mixing of isotopes among specified atom positions in an ion or neutral species.

Note: In mass spectrometry, this term usually refers to the partial random mixing of isotopes in isolated ions that were formed with a fixed isotopic composition.

Revised from [5,6] using additional information from [255].

\section{4. particle beam interface}

Method for coupling liquid chromatography to mass spectrometry in which the effluent is passed through a heated capillary to form an expanding jet of vapour and aerosol particles. After passing through a skimmer that acts as a momentum separator, the beam impinges on a heated surface to form ions through chemical ionization at the surface or ionization of the resulting vapour in a chemical ionization or electron ionization source.

From [256,257]. 


\section{Paul ion trap \\ quadrupole ion trap \\ quadrupole ion storage trap}

Ion trapping device that depends on the application of radio frequency potentials between a ring electrode and two end-cap electrodes to confine the ion motion to a cyclic path described by an appropriate form of the Mathieu equation. The choice of these potentials determines the $m / z$ value below which ions are not trapped.

From $[10,52,62]$.

386. peak (in mass spectrometry)

See mass peak.

\section{7. peak height}

Height of a recorded peak in a mass spectrum.

From [5].

\section{8. peak matching}

Procedure for measuring the accurate mass of an ion using scanning mass spectrometers, in which the peak corresponding to the unknown ion and that for a reference ion of known $\mathrm{m} / \mathrm{z}$ are displayed alternately and caused to overlap by adjusting appropriate electric fields.

From [258].

\section{9. peak parking}

Method used to extend the amount of time available for mass spectrometric analysis of the components in a narrow chromatographic peak. The eluent flow rate is reduced to an ultra-low level at the leading edge of the peak and held at this low flow rate for the duration of compound elution, thus enabling collection of sufficient data for reliable statistical analysis.

From [259].

\section{0. peak stripping}

Data analysis technique used in elemental mass spectrometry to correct the intensity of an unresolved multiplet peak monitored for a specific element for contributions from a multi-isotopic contaminant. The correction is calculated from the measured intensity of a different isotopic peak of the contaminant and the known natural isotopic abundance ratio.

From [260].

\section{Penning ionization}

Ionization via electron removal that occurs through the energy transfer from a neutral species $(\mathrm{N})$ internally excited to a metastable state (such as a high Rydberg state) to a neutral species (M) with lower ionization energy, as represented by the following reaction: $\mathrm{N}^{*}+\mathrm{M} \rightarrow \mathrm{N}+\mathrm{M}^{+\bullet}+e^{-}$.

Note: Penning ionization differs from chemi-ionization in that no chemical change is involved.

From [5]. 


\section{Penning ion trap}

Ion trapping device using a static magnetic field and a static electric potential well. The magnetic field confines the ion motion to circular orbits around the magnetic force line direction, which aligns with the direction of the trap axis, whereas the electric potential well confines the ion motion axially.

Note: $\quad$ This device is used for the mass analyzer of Fourier transform-ion cyclotron resonancemass spectrometers.

From [261].

\section{3. peptide mass fingerprinting (PMF) mass mapping}

Method for protein analysis where an unknown protein is chemically or enzymatically cleaved into peptide fragments whose masses are determined by mass spectrometry. The peptide masses are compared to peptide masses calculated for known proteins in a database and analyzed statistically to determine the best match.

See also accurate mass tag.

From [262].

\section{4. peptide sequence tag}

Sequence of peptide ion fragment masses that can be used to aid in the identification of the amino acid sequence.

Note: $\quad$ Sequence tags are often obtained in tandem mass spectrometry analysis of peptides from an unknown protein that has been chemically or enzymatically cleaved into peptide fragments.

From [263].

\section{5. photodissociation}

Process wherein the reactant ion or molecule is dissociated as a result of absorption of one or more photons.

From [264].

\section{6. photographic plate recording}

Recording of ion currents by allowing them to strike a photographic plate, which is subsequently developed.

Note: $\quad$ This technique is typically associated with ion-beams that have been spatially separated by $\mathrm{m} / \mathrm{z}$ and focused across a focal plane as in a Mattauch-Herzog geometry magnetic sector instrument.

From [5].

\section{7. photoionization (PI)}

Deprecated: photon impact.

Ionization of an atom or molecule by a photon, written $\mathrm{M}+h v \rightarrow \mathrm{M}^{+\bullet}+e^{-}$.

See also multiphoton ionization.

From $[5,6]$. 


\section{8. photon impact}

This term is deprecated.

See photoionization.

\section{9. $\pi / n \operatorname{rad}\left(180^{\circ} / n\right)$ magnetic sector}

Arrangement in which an ion beam is deflected magnetically through $\pi / n \operatorname{rad}$, where $n>1$. From [5].

400. plasma (in spectrochemistry)

Gas that is at least partly ionized and contains particles of various types: electrons, atoms, ions, and molecules. The plasma as a whole is electrically neutral.

From $[5,6]$.

\section{1. plasma desorption ionization (PDI)} fission fragment ionization

Ionization of material in a solid sample by bombarding it with ions and/or neutral atoms formed as a result of the fission of a suitable nuclide, typically ${ }^{252} \mathrm{Cf}$.

From $[5,6]$.

\section{2. pneumatically assisted electrospray ionization}

Electrospray ionization in which the nebulization of the liquid stream is assisted by a concentric stream of gas.

Note: Ionspray should only be used to describe the commercial product. From [265].

\section{3. point detector}

Detector in which the ion beam is focused onto a small area, for example, defined by a collector slit, rather than dispersed by $\mathrm{m} / \mathrm{z}$ spatially across a focal plane.

From [266].

\section{4. positive ion}

Atomic or molecular species having a net positive electric charge; a cation.

From $[5,6]$.

\section{5. post-acceleration detector (PAD)}

Detector in which a high potential is applied after $\mathrm{m} / \mathrm{z}$ separation to accelerate the ions and produce an improved signal.

See also conversion dynode.

From [267]. 


\section{6. post-source decay (PSD)}

Technique specific to reflectron time-of-flight mass spectrometers where product ions of metastable transitions or collision-induced dissociations generated in the flight tube prior to entering the reflectron are separated according to $\mathrm{m} / \mathrm{z}$ to yield product ion spectra.

From [268].

\section{7. precursor ion} progenitor ion

Deprecated: parent ion.

Ion that reacts to form particular product ions or undergoes specified neutral losses. The reaction can be of different types including unimolecular dissociation, ion/molecule reaction, change in charge state, possibly preceded by isomerization.

Revised from $[5,6]$ using additional information from $[8,16,17]$.

\section{8. precursor ion spectrum}

Deprecated: parent ion spectrum.

Mass spectrum in which the appropriate $\mathrm{m} / \mathrm{z}$ separation function records the precursor ions of selected product ions or specified neutral loss.

Note: The ion current recorded by the detector is that from the product ion but the $m / z$ values corresponding to the peaks in the resulting spectrum are those of precursor ions that fragmented in the fashion specified by the $\mathrm{m} / \mathrm{z}$ separation function of the tandem mass spectrometer.

See also product ion spectrum.

From $[8,16,17]$.

\section{9. pre-ionization state}

Electronic state capable of undergoing autoionization.

From $[5,6]$.

\section{0. principal ion}

Most abundant ion of an isotope cluster.

Note 1: An example is the ${ }^{11} \mathrm{~B}^{79} \mathrm{Br}_{2}{ }^{81} \mathrm{Br}^{+\bullet}$ ion of $m / z 250$ of the cluster of isotopolog molecular ions of $\mathrm{BBr}_{3}$.

Note 2: Principal ion has also been used to describe ions that have been artificially isotopically enriched in one or more positions such as ${ }^{13} \mathrm{CH}_{3}{ }^{+\bullet}$ or $\mathrm{CH}_{2} \mathrm{D}_{2}{ }^{+}$, but those are best defined as isotopolog ions.

From $[5,6]$.

\section{1. probability-based matching}

Automated system for computer examination of the mass spectrum of an unknown mixture for the presence of a specific compound for which a standard spectrum is available in a library. The probability that the compound is present is given by a confidence index $K$ such that $2^{K}$ is the average number of ran- 
domly selected compounds whose mass spectra would have to be examined to find data which match the target spectrum to the same degree as does the unknown.

From [269].

\section{2. product ion}

Deprecated: daughter ion.

Ion formed as the product of a reaction involving a particular precursor ion.

Note: The reaction can be of different types including unimolecular dissociation to form fragment ions, an ion/molecule reaction, or simply involve a change in the number of charges.

Revised from [5,6] using additional information from $[8,16,17]$.

\section{3. product ion analysis}

Deprecated: daughter ion analysis.

Process whereby a precursor ion of a particular $\mathrm{m} / \mathrm{z}$ is selected by $\mathrm{m} / \mathrm{z}$, fragmentation is induced, and the mass spectrum of the resulting product ions is recorded.

Revised from [5] using additional information from [152,153].

\section{4. product ion spectrum}

Mass spectrum in which the appropriate $\mathrm{m} / \mathrm{z}$ separation analysis function is set to record the product ions of a selected precursor ion selected by $\mathrm{m} / \mathrm{z}$.

See also precursor ion spectrum.

From $[8,16,17]$.

\section{5. profile mode}

Method for acquiring a mass spectrum where each peak is displayed as a curve, with the data points defining the curve corresponding to the signal intensities at each particular $m / z$ value.

See also centroid acquisition.

From [270,271].

\section{6. progenitor ion}

See precursor ion.

From $[5,6]$.

\section{7. prolate trochoidal mass spectrometer}

Deprecated: cycloidal mass spectrometer.

Mass spectrometer in which the ions of different $\mathrm{m} / \mathrm{z}$ are separated by means of crossed electric and magnetic fields in such a way that the selected ions follow a prolate trochoidal path.

From $[5,6]$. 


\section{8. proton affinity (PA)}

For a species $M$, the negative of the enthalpy change for the gas phase reaction $\mathrm{M}+\mathrm{H}^{+} \rightarrow[\mathrm{M}+\mathrm{H}]^{+}$at a specified temperature, usually $298 \mathrm{~K}$.

From $[5,6]$.

\section{9. protonated molecular ion}

This term is deprecated.

See protonated molecule.

\section{0. protonated molecule}

Deprecated: protonated molecular ion, pseudo-molecular ion, quasi-molecular ion.

Adduct ion, represented by $[\mathrm{M}+\mathrm{H}]^{+}$, formed by the interaction of a molecule with a proton (hydron).

Note 1: Multiply protonated molecules are represented by $[\mathrm{M}+n \mathrm{H}]^{n+}$ where $n$ is the number of protons.

Note 2: Protonated molecular ion is deprecated; for example, for a molecular ion $\mathrm{M}^{+\bullet}$ this would correspond to a species carrying two positive charges plus an unpaired electron.

Note 3: Pseudo-molecular ion and quasi-molecular ion are deprecated; a specific term such as protonated molecule, or a chemical description such as $[\mathrm{M}+\mathrm{H}]^{+}$should be used.

See also adduct ion.

Revised from [5,6] using additional information from [16].

\section{1. proton-bound dimer}

Dimeric ion consisting of two similar or dissimilar molecules bound together by hydrogen bonding with a shared proton.

From $[272,273]$.

\section{2. pseudo-molecular ion}

This term is deprecated.

See protonated molecule.

\section{3. pyrolysis mass spectrometry (PyMS)}

Mass spectrometry technique in which the sample is heated to the point of decomposition and the gasphase decomposition products are characterized by mass spectrometry.

From [274].

\section{4. quadratic field reflectron}

Reflectron in which the electric field varies with the square of the distance from the entrance and compensates for kinetic energy spread to all orders.

From [275]. 


\section{5. quadrupolar axialization}

Technique for axialization in Fourier transform-ion cyclotron resonance-mass spectrometers in which magnetron motion is converted into cyclotron motion by subjecting the trapped ions to a quadrupolar excitation field while they undergo collisions.

From [276,277].

\section{6. quadrupole ion storage trap (QUISTOR)}

See Paul ion trap.

\section{7. quadrupole ion trap (QIT)}

See Paul ion trap.

\section{8. quadrupole mass analyzer}

See transmission quadrupole mass spectrometer.

\section{9. quadrupole mass filter (QMF)}

See transmission quadrupole mass spectrometer.

\section{0. quadrupole mass spectrometer (QMS)}

See transmission quadrupole mass spectrometer.

\section{1. quadrupole time-of-flight (QTOF)}

Hybrid mass spectrometer consisting of a transmission quadrupole mass spectrometer coupled to an orthogonal acceleration time-of-flight mass spectrometer. A collision quadrupole is typically inserted between the two mass spectrometers.

From [278].

\section{2. quantitation by concatenated tryptic peptides (QCAT)}

Method for absolute quantitation of a protein or proteins in a multiplexed fashion using isotope-labeled peptides as surrogate internal standards. The labeled peptides are produced by synthesizing a gene coding for all desired peptides, expressing this gene in E. coli culture containing appropriately labeled substrate, and digesting with trypsin the expressed protein composed of the desired concatenated peptides. From [279].

\section{3. quasi-equilibrium theory (QET)}

Statistical theory of unimolecular reaction rates (isomerization or fragmentation) of molecular species that assumes that reaction occurs from the ground electronic state of the reactant regardless of its mode of formation, at a rate determined by the free statistical redistribution of internal energy into those vibrational and rotational modes that must be activated sufficiently to reach the appropriate transition state for the specified reaction to occur. The QET applies to isolated molecular species in the absence of collisions, as for ion fragmentations in a mass spectrometer. Early formulations assumed the reactant con- 
tained $s$ identical harmonic oscillators of frequency $v$, with a transition state corresponding to just one critical oscillator containing at least $m$ quanta corresponding to the critical energy for reaction $\varepsilon^{*}=m v$. A further assumption that $m>s$ leads to an ultra-simple expression for the unimolecular rate constant at total internal energy $\varepsilon$ : $k(\varepsilon)=v\left[\left(\varepsilon-\varepsilon^{*}\right) / \varepsilon\right]^{(s-1)}$. Elimination of most of these simplifying assumptions followed development of the Rice-Ramsperger-Kassel-Marcus (RRKM) theory.

See also Rice-Ramsperger-Kassel (RRK) theory.

Revised from [6] using additional information from [280,281].

\section{4. quasi-molecular ion}

This term is deprecated.

See protonated molecule.

\section{5. radial ejection}

Ejection of an ion from an ion trap in a direction perpendicular to the longitudinal axis of the trap. From [282].

\section{6. radial electrostatic field analyzer}

Arrangement of two conducting sheets forming a capacitor and giving a radial electrostatic field which is used to deflect and focus ion beams. The degree of deflection varies with the ratio of translational energy to charge. The capacitor may be cylindrical, spherical, or toroidal.

Revised from [5,6].

\section{7. radical ion odd-electron ion}

Cation or anion containing unpaired electrons in its ground state. An unpaired electron is denoted by a superscript dot following the superscript symbol for charge, such as for the molecular ion of a molecule $\mathrm{M}$, that is, $\mathrm{M}^{+\bullet}$. Radical ions with more than one charge and/or more than one unpaired electron are denoted using parentheses, for example, as $\mathrm{M}^{(2+)(2 \bullet)}$.

Note 1: Unless the positions of the unpaired electron and charge can be associated with specific atoms, superscript charge designation should be placed before the superscript dot designation.

Note 2: The order of the unpaired electron and charge are reversed from that recommended for organic and inorganic chemistry in which the dot representing the unpaired electron precedes the symbol for the charge. This convention is widely used in the mass spectrometry field and has been in place for many decades, and is retained here for that reason.

Note 3: It is not recommended that the charge designation be placed directly above the centrally placed dot because of the difficulty of extending it to ions bearing more than one charge and/or more than one unpaired electron.

From [6]. 


\section{Rayleigh limit}

Limiting droplet size at which self-fragmentation will occur for charged droplets generated in electrospray or other ionization processes. Self-fragmentation occurs when the electrostatic repulsion force generated by the excess charge exceeds the surface tension that maintains the integrity of the droplet. From [283].

\section{9. reagent gas}

Gas that reacts with ions to produce product ions through ion/molecule reactions; for example, a gas such as methane, ammonia, or isobutane used in chemical ionization to create reagent ions or a gas used in ion traps or collision cells to perform gas-phase ion/molecule reactions.

From [284].

\section{0. reagent ion}

Ion that reacts with a neutral to produce an ionized form of such species through an ion/molecule reaction, for example, as in chemical ionization.

From [284].

\section{1. rearrangement ion}

Ion that results from molecular rearrangement; in which its atoms or groups of atoms have transferred from one portion of the molecule to another during or following ionization.

From $[5,6]$.

\section{2. recombination energy}

Energy released when an electron is added to an ionized molecule or atom.

Note: This is the energy required for the reverse process of vertical ionization. From [285].

\section{3. reconstructed ion chromatogram}

See extracted ion chromatogram.

\section{4. reconstructed total ion current chromatogram}

See total ion current chromatogram.

\section{5. rectilinear ion trap}

Quadrupole ion trap whose electrodes form a rectilinear polygon, the simplest form of which consists of two pairs of rectangular electrodes supplied with radio frequency (RF) potentials and a pair of electrodes to which a DC potential is applied. Slits or apertures in the electrodes allow injection and ejection of charged particles.

From [286]. 


\section{6. reference ion}

Stable ion whose structure or elemental formula is known with certainty. These ions are formed by direct ionization of a molecule of known structure or elemental formula, and are used to verify by comparison the structure or thermochemistry of an unknown ion or to calibrate the $\mathrm{m} / \mathrm{z}$ scale of the mass spectrometer.

From [287-289].

\section{7. reflectron}

Component of a time-of-flight mass spectrometer that uses a static electric field to reverse the direction of travel of the ions and improves mass resolution by assuring that ions of the same $\mathrm{m} / \mathrm{z}$ but different translational energy arrive at the detector at the same time.

From [290].

\section{8. relative detection limit detection limit}

Smallest amount of material detectable in a matrix relative to the amount of material analyzed, given in atomic, mole, or weight fractions.

Note 1: The meaning of "detectable" must be specified, for example, three times the standard deviation of the measurement ( $3 \sigma$ criterion).

Note 2: Often incorrectly referred to as sensitivity. From [6]

\section{9. relative difference of isotope ratios}

See relative isotope-ratio difference.

450. relative intensity (in mass spectrometry)

See intensity relative to base peak (in mass spectrometry).

From [8].

451. relative isotope-ratio difference, $\delta$

delta notation

isotope delta

relative difference of isotope ratios

Measure of an isotope ratio in a sample of interest relative to a reference defined by the relation

$$
\delta=\left(R_{\text {sample }}-R_{\text {standard }}\right) / R_{\text {standard }}
$$

where $R$ is the isotope ratio of the element.

Note 1: The reference is often an international measurement standard.

Note 2: The same relative differences are used to calculate delta values from the abundance of isotopomers and isotopologs, ion currents, frequencies, or optical extinctions.

Note 3: Relative isotope ratio differences are usually small and are therefore often expressed in units of parts per mil with symbol \%o. 
Note 4: Units of parts per thousand (ppt, $10^{-3}$ ) or parts per million (ppm, $10^{-6}$ ) are deprecated [7].

From $[196,197]$.

\section{2. residual gas analyzer (RGA)}

Mass spectrometer used to measure the composition and pressure of gases in an evacuated chamber and to test for leaks in pressurized systems.

From [291].

\section{3. residual spectrum}

Mass spectrum with peaks recorded in the absence of sample and due either to small air leaks or to the presence of molecules desorbed from the walls of the introduction device, the ion source, or from vacuum pump fluids.

See also background mass spectrum.

From [11].

\section{4. resolution (in mass spectrometry)} mass resolution

In a mass spectrum, the observed $\mathrm{m} / \mathrm{z}$ value divided by the smallest difference $\Delta(\mathrm{m} / \mathrm{z})$ for two ions that can be separated: $(\mathrm{m} / \mathrm{z}) / \Delta(\mathrm{m} / \mathrm{z})$.

Note 1: The $m / z$ value at which the measurement was made should be reported.

Note 2: The definition and method of measurement of $\Delta(\mathrm{m} / z)$ should be reported. Commonly this is performed using peak width measured at a specified percentage of peak height.

Note 3: Alternatively $\Delta(\mathrm{m} / \mathrm{z})$ is defined as the separation between two adjacent equal magnitude peaks such that the valley between them is a specified fraction of the peak height, for example as measured by peak matching.

See also resolving power (in mass spectrometry).

Revised from [5,10] using additional information from [292,293].

\section{5. resolution: 10 per cent valley definition}

Value of $(\mathrm{m} / \mathrm{z}) / \Delta(\mathrm{m} / \mathrm{z})$ measured for two peaks of equal height in a mass spectrum at $\mathrm{m} / \mathrm{z}$ and $\mathrm{m} / \mathrm{z} \pm$ $\Delta(\mathrm{m} / \mathrm{z})$ that are separated by a valley which at its lowest point is $10 \%$ of the height of either peak. For peaks of similar height separated by a valley, let the height of the valley at its lowest point be $10 \%$ of the lower peak. Then the resolution (10\% valley definition) is $(\mathrm{m} / \mathrm{z}) / \Delta(\mathrm{m} / \mathrm{z})$ and should be given for a number of values of $m / z$.

See also resolution: peak width definition.

From $[5,6]$.

\section{6. resolution: peak width definition}

For a single peak corresponding to singly charged ions at mass $m$ in a mass spectrum, the resolution may be expressed as $(\mathrm{m} / \mathrm{z}) / \Delta(\mathrm{m} / \mathrm{z})$, where $\Delta(\mathrm{m} / \mathrm{z})$ is the width of the peak at a height which is a specified fraction of the maximum peak height. It is recommended that one of three values 50,5 , or $0.5 \%$ should always be used. 
Note: For an isolated symmetrical peak recorded with a system which is linear in the range between $5 \%$ and $10 \%$ levels of the peak, the $5 \%$ peak width definition is equivalent to the $10 \%$ valley definition. A common standard is the definition of resolution based upon $\Delta m$ being full width of the peak at half its maximum (FWHM) height.

See also resolution: 10 per cent valley definition.

From $[5,6]$.

457. resolving power (in mass spectrometry) mass resolving power

Measure of the ability of a mass spectrometer to provide a specified value of mass resolution.

Note: $\quad$ The procedure by which $\Delta(\mathrm{m} / \mathrm{z}$ ) was defined and measured, and the $\mathrm{m} / \mathrm{z}$ value at which the measurement was made, should be reported.

Revised from $[5,6]$.

\section{8. resonance-enhanced multiphoton ionization (REMPI) resonance ionization}

Multiphoton ionization in which the ionization cross section is significantly enhanced because the energy of the incident photons is resonant with an intermediate excited state of the neutral.

From [294,295].

\section{9. resonance ion ejection}

Mode of ion ejection in an ion trap that relies on an auxiliary radio frequency potential that is applied to the end-cap or trapping electrodes. The potential is tuned to the classical oscillation frequency of a particular ion to eject it.

From [296].

\section{0. resonance ionization $(\mathrm{RI})$}

See resonance-enhanced multiphoton ionization.

\section{1. retro Diels-Alder reaction}

Ion fragmentation mechanism in which the molecular ion $\mathrm{M}^{+\bullet}$ of a cyclic alkene fragments to form a neutral diene and an alkene radical cation or a diene radical cation and an alkene. The reaction is the reverse of the Diels-Alder reaction of organic synthesis.

From [297].

\section{2. reverse geometry}

Double-focusing mass spectrometer configuration in which the ion beam traverses the magnetic sector prior to the electric sector.

See also forward geometry.

From [149]. 


\section{3. reverse library search}

Process of comparing a subset of the peaks in the mass spectrum of an unknown compound with peaks present in the library mass spectrum. Any peaks in the mass spectrum of the unknown compound that are not in the library spectrum are disregarded.

Note: A reverse library search measures the extent to which the peaks in a reference spectrum are present in the unknown spectrum and is more effective than a forward library search when peaks arising from two or more components are present in the unknown spectrum

See also forward library search.

From [230].

\section{RF-DC ion mobility spectrometry}

See high-field asymmetric waveform ion mobility spectrometry.

\section{RF-only quadrupole}

Transmission quadrupole device in which a sinusoidal radio frequency (RF) potential is applied between the connected pairs of opposite rod electrodes in a linear quadrupole, providing a transmission device for all ions with $\mathrm{m} / \mathrm{z}$ values above a cut-off value.

Note: Higher-order multipoles can also be used in this manner.

See also collision quadrupole.

From [78].

\section{Rice-Ramsperger-Kassel-Marcus (RRKM) theory}

Statistical theory of unimolecular reaction rates of gas-phase molecular species that removes most of the simplifying assumptions in the Rice-Ramsperger-Kassel (RRK) theory. Account is taken of the way in which the different normal-mode vibrations and rotations contribute to reaction, and allowance is made for the zero-point energies. The total internal energy is partitioned into active and inactive components, such that only the active component can flow freely among the internal modes and thus contribute to reaction. The assumed equilibrium ratio of active-to-inactive components is evaluated using partition functions. In applications where gas-phase collisions are important, the rates of activation and deactivation take into account their energy dependence, and in the high-pressure limit the transition state is in equilibrium with non-activated reactants and RRKM reduces to conventional transition-state theory.

Note: The theory is also used (with some modification) for reactions in condensed phases. Revised from [6] using additional information from [298-300].

\section{Rice-Ramsperger-Kassel (RRK) theory}

Statistical theory of unimolecular reaction rates of gas-phase molecular species that is equivalent to the quasi-equilibrium theory, but with the added consideration that the effects of gas-phase collisions in activation to and deactivation from the transition state are taken into account.

Note: Elimination of most of the simplifying assumptions led to development of the RiceRamsperger-Kassel-Marcus (RRKM) theory.

Revised from [6] using additional information from [281,301,302]. 


\section{8. ring and double bond equivalent}

Deprecated: rings plus double bonds.

Conventional measure of the degree of unsaturation of an organic molecule given by $X-Y / 2+$ $Z / 2+1$ where $X$ is the number of carbon atoms, $Y$ is the number of hydrogen or halogen atoms and $Z$ is the number of nitrogen and phosphorus atoms.

From [24,303].

\section{9. rings plus double bonds}

This term is deprecated.

See ring and double bond equivalent.

\section{0. saddle field gun}

Compact ion and fast atom sources for use when small size and high beam intensity are required, as in fast atom bombardment mass spectrometry. The discharge is produced by electrons oscillating between two cold cathodes in an electric field that possesses a single saddle point. To produce and intensify the plasma the electrons must have long path lengths, achieved by allowing the electrons to have an oscillatory motion in the saddle-shaped electric field between the cathodes separated by the anode.

From [304].

\section{1. sampling cone}

Component of a spray ionization interface that consists of a cone with a small orifice through which the spray passes into a differentially pumped chamber

Note 1: In interfaces that have both a sampling cone and skimmer, the spray passes first through the sampling cone and then through the skimmer.

Note 2: A capillary can also be used in place of a cone. From $[49,254,305]$.

\section{2. scan cycle time}

Time required to obtain a mass spectrum (or other mass spectrometric data quantity) such as in a separation coupled to mass spectrometry.

From $[137,306]$.

\section{3. secondary electron}

Electrons ejected from a sample surface as a result of bombardment by a primary beam of atoms, ions, electrons, or photons.

From [307].

\section{4. secondary ionization}

Process in which ions are ejected from a sample surface as a result of bombardment by a primary beam of atoms or ions.

See also secondary ion mass spectrometry (SIMS).

From [6]. 


\section{5. secondary ion mass spectrometry (SIMS)}

Technique in which a focused beam of primary ions produces secondary ions by sputtering from a solid surface. The secondary ions are analyzed by mass spectrometry.

See also dynamic secondary ion mass spectrometry (DSIMS), static secondary ion mass spectrometry (SSIMS).

From [308].

\section{6. secondary neutral mass spectrometry (SNMS)}

Mass spectrometry technique in which neutral species ejected from a sample surface as a result of bombardment by a primary beam of atoms or ions are ionized, most often by photoionization, prior to analysis.

See also secondary ion mass spectrometry (SIMS).

From $[309,310]$.

\section{7. sector mass spectrometer}

Mass spectrometer consisting of one or more magnetic sectors for recording a mass spectrum of a monoenergetic beam of ions. Such instruments may also have one or more electric sectors for energy dispersion that can be arranged for velocity-focusing by compensating for dispersion by the magnetic sector if the beam is not truly monoenergetic. Such a combination provides double focusing of ions of the same $m / z$, i.e., focusing with respect to both direction and speed.

From [311].

\section{8. selected ion flow tube (SIFT)}

Device in which $\mathrm{m} / \mathrm{z}$ selected ions are entrained in an inert carrier gas and subsequently undergo ion/molecule reactions with molecules introduced into the gas flow.

See also drift tube.

From [312].

\section{9. selected ion monitoring (SIM)}

Deprecated: multiple ion detection, mass fragmentography, selected ion recording.

Operation of a mass spectrometer in which the abundances of ions of one or more specific $\mathrm{m} / \mathrm{z}$ values are recorded rather than the entire mass spectrum.

See also selected reaction monitoring (SRM).

Revised from [5,6] using additional information from [313].

\section{0. selected ion recording (SIR)}

This term is deprecated.

See selected ion monitoring (SIM).

\section{1. selected reaction monitoring (SRM)}

Data acquired from one or more specific product ions corresponding to $\mathrm{m} / \mathrm{z}$ selected precursor ions recorded via two or more stages of mass spectrometry. 
Note 1: Selected reaction monitoring in multiple-stage mass spectrometry is known as consecutive reaction monitoring.

Note 2: Selected reaction monitoring applied to multiple product ions from one or more precursor ions is known as multiple reaction monitoring.

See also selected ion monitoring (SIM).

From [314].

\section{2. self-chemical ionization (self-CI)}

Chemical ionization of an analyte where the reagent ion is an ionized form of the analyte or one of its product ions.

From [315].

\section{3. sensitivity, $A$}

Slope of the calibration curve.

Note 1: If the curve is not a straight line, sensitivity will be a function of analyte concentration or amount.

Note 2: If sensitivity is to be a unique performance characteristic, it must depend only on the From $[5,6]$. chemical measurement process, not upon scale factors.

\section{4. sheath flow interface}

Capillary electrophoresis to electrospray ionization interface that uses a coaxial flow of make-up liquid that is introduced through a tube that is concentric with the separation capillary.

From [216,217].

\section{5. sheath gas}

Gas introduced through a tube that is coaxial with the electrospray emitter to pneumatically assist the formation of the sprayed droplets.

From [316].

\section{6. sheath liquid}

Make-up liquid in a sheath flow interface.

From [26].

\section{7. shotgun proteomics}

Protein identification using a combination of high-performance liquid chromatography and mass spectrometry in which the proteins in a mixture are digested, usually by a specific enzyme, and the resulting peptides are separated by liquid chromatography and identified by tandem mass spectrometry.

See also bottom-up proteomics.

From [317]. 


\section{8. skimmer}

Cone with a central orifice that intercepts the center of a spray or jet expansion to sample the central portion of the expansion, for example, as in a momentum separator.

From [253].

\section{9. soft ionization}

Formation of gas-phase ions without extensive fragmentation.

See also hard ionization.

From [318].

\section{0. sonic spray ionization (SSI)}

Process in which both anions and cations are produced at atmospheric pressure from an ionic solution due to a statistically unbalanced charge distribution in the minute droplets formed by the action of a sonic atomizer.

See also spray ionization.

From [319].

\section{1. space charge effect}

Result of mutual repulsion of particles of like charge (ions, electrons, etc.) that can limit the current in a charged-particle beam and can also cause some motion of ions in a beam or packet in addition to that caused by external fields.

Note: $\quad$ Space charge effects on ion trajectories are most noticeable in ion trap instruments that confine large numbers of ions in a small volume.

From [320].

\section{2. spark ionization}

Formation of ions from a solid material by an intermittent electric discharge.

From $[5,6]$.

\section{3. spectral skewing}

Deviation of the peak intensities in a mass spectrum from their expected values that occurs when the relative concentration of the various analytes change during acquisition of the mass spectrum.

From [321].

\section{4. spray ionization}

General term used to describe a range of ionization techniques for analytes in solution that involve dispersing the liquid into fine droplets as a component step.

Note: $\quad$ Most of these techniques are examples of atmospheric pressure ionization.

From [322]. 


\section{5. stable ion}

Ion with internal energy sufficiently low that it does not rearrange or dissociate prior to detection in a mass spectrometer.

From $[5,6]$.

\section{6. stable isotope mass spectrometry}

See isotope ratio mass spectrometry.

\section{7. stable isotope ratio analysis of amino acids in cell culture (SILAC)}

Proteomics technique that detects differences in protein abundance between cultured cell samples using stable isotopic labeling achieved with labeled amino acids in one cell culture and unlabeled ones in a separate culture.

From [323-325].

\section{8. stable isotope standards and capture by anti-peptide antibodies (SISCAPA)}

Method for absolute quantitation of a protein or proteins in a multiplexed fashion using isotope-labeled peptides as surrogate internal standards. The labeled peptides are produced by synthesizing a gene coding for each desired peptide, cell-free transcription, and translation of this gene in a medium containing ${ }^{13} \mathrm{C}_{6}$-labeled lysine, and digesting with trypsin the expressed protein composed of the desired concatenated peptides. For low-abundance proteins the sensitivity is increased by selective enrichment of the target peptides by antibody capture.

From [326].

\section{9. stability diagram}

See Mathieu stability diagram.

\section{0. static field}

Electric or magnetic field that does not change in time.

From [327].

\section{1. static secondary ion mass spectrometry (SSIMS)}

Method of secondary ion mass spectrometry using low current densities for analysis of sample surface components, in contrast with dynamic secondary ion mass spectrometry. which is used for analysis of components in the depth direction.

Note: When the sample is an organic molecule in solid form the term is often changed to From [124]. organic SIMS or molecular SIMS.

\section{Stevenson's rule}

Rule stating that in competing fragmentations the product ion formed from its neutral species counterpart with the lower ionization energy will usually be the more abundant. 
Note: The original version of this rule referred to reliability of determination of thermochemical data obtained for an ion produced by fragmentation of a single $\sigma$-bond in an oddelectron ion $\mathrm{X}_{-} \mathrm{Y}^{+}$, but was later extended to the version in more common use since then.

From [328-330].

\section{3. stored waveform inverse Fourier transform (SWIFT)}

Technique to create excitation waveforms for ions in Fourier transform-ion cyclotron resonance-mass spectrometers or Paul ion traps. An excitation waveform in the time-domain is generated by taking the inverse Fourier transform of an appropriate frequency-domain programmed excitation spectrum, in which the resonance frequencies of ions to be excited are included.

Note: This procedure may be used for selection of precursor ions in $M S^{n}$ experiments. From [331].

504. supercritical fluid chromatography-mass spectrometry (SFC-MS) supercritical fluid chromatography/mass spectrometry (SFC/MS)

Combined technique in which a mixture of analytes is separated into solutions of individual components by chromatography that uses a supercritical fluid as mobile phase, followed by the ionization of the separation effluent in the ion source of a mass spectrometer.

From [332].

\section{5. surface-assisted laser desorption/ionization (SALDI)}

Class of matrix-free laser desorption ionization techniques for biological macromolecules.

Note: $\quad$ An example is desorption ionization on silicon (DIOS). From [333].

\section{6. surface-enhanced affinity capture (SEAC)}

See surface-enhanced laser desorption/ionization (SELDI)

\section{7. surface-enhanced laser desorption/ionization (SELDI)}

Variant of matrix-assisted laser desorption/ionization (MALDI) in which the matrix is replaced by a surface coating of a chromatographic stationary phase designed to selectively retain different subsets of proteins or peptides from a mixture depending on their physico-chemical properties or biochemical affinity characteristics.

Note: Originally this technique was named surface-enhanced affinity capture (SEAC) to distinguish it from surface-enhanced neat desorption (SEND) where the surface coating is From [334,335]. designed to increase the efficiency of laser desorption.

508. surface-enhanced neat desorption (SEND)

See surface-enhanced laser desorption/ionization (SELDI). 


\section{9. surface-induced dissociation (SID)}

Fragmentation that results from the collision of an ion with a surface. From [336].

\section{0. surface-induced reaction (SIR)}

Process wherein a reactant ion interacts with a surface to produce either chemically different species or a change in the internal energy of the reactant ion.

From [337].

\section{1. surface ionization (SI)}

Ionization of a neutral when it interacts with a solid surface with an appropriate work function and temperature.

From $[5,6]$.

\section{2. surrogate internal standard internal standard}

Compound added in accurately known amounts to a sample to be analyzed for a specified analyte. The surrogate internal standard is chemically similar to the analyte so that losses in the analytical procedure are the same for both and thus the analyte concentration can be determined relative to that of the standard.

Note 1: In quantitative mass spectrometry the preferred type is an isotope-labeled version of the analyte.

Note 2: The abbreviated term internal standard is often used.

See also volumetric internal standard.

From [338].

\section{3. sustained off-resonance irradiation (SORI)}

Procedure associated with Fourier transform-ion cyclotron resonance-mass spectrometers to facilitate ion/neutral reactions such as low-energy collision-induced dissociation in which a radio-frequency electric field, slightly off-resonance with respect to the cyclotron frequency of the reactant ion, cyclically accelerates and decelerates the reactant ion that is confined in the Penning ion trap. As a consequence, the orbit does not exceed the dimensions of the ion trap but maintains a high average translational energy for an extended time that activates an ion/neutral process.

From [339].

\section{4. tandem mass spectrometer}

Mass spectrometer designed for mass spectrometry/mass spectrometry.

From $[5,6]$.

\section{5. tandem mass spectrometry}

See mass spectrometry/mass spectrometry (MS/MS). 


\section{6. tandem mass spectrometry in space}

Tandem mass spectrometry procedure in which product ion spectra are recorded for ion beams in $\mathrm{m} / \mathrm{z}$ analyzers separated in space. Specific $m / z$ separation functions are designed so that in one section of the instrument ions are selected, dissociated in an intermediate region, and the product ions are then transmitted to another analyzer for $m / z$ separation and data acquisition.

From [340].

\section{7. tandem mass spectrometry in time}

Tandem mass spectrometry procedure in which product ion spectra are recorded in a single $\mathrm{m} / \mathrm{z}$ analyzer (such as a Paul ion trap, linear ion trap, or Fourier transform-ion cyclotron resonance-mass spectrometer) in discrete steps over time. Ions in a specific $\mathrm{m} / \mathrm{z}$ range are selected, dissociated, and the product ions analyzed sequentially in time.

Note: Mass spectrometers that can perform tandem mass spectrometry in time can also perform $M S^{n}$.

From [340].

\section{8. target gas}

This term is deprecated.

See collision gas.

\section{Taylor cone}

Conical shape of the liquid emanating from a capillary under high potential, as in electrospray or other electrohydrodynamic spray processes.

From [341,342].

\section{0. thermal ionization (TI)}

Ionization of a neutral through contact with a high-temperature surface.

From $[5,6]$.

\section{1. thermospray ionization}

Spray ionization in which a liquid is flowed through a heated capillary to produce a spray of droplets and solvent vapor. Ions are formed due to the statistical imbalance of charges in the droplets or by a heated filament.

From $[343,344]$.

\section{2. thin layer chromatography-mass spectrometry (TLC-MS) thin layer chromatography/mass spectrometry (TLC/MS)}

Technique by which a mixture of analytes is separated into individual components by liquid chromatography, followed by the ionization of the separated species by an ion source of a mass spectrometer.

From [345]. 


\section{3. thomson, $T h$}

This term is deprecated.

See $m / z$.

\section{4. time-lag focusing (TLF)}

Energy focusing of ions formed in the gas phase in a time-of-flight mass spectrometer that is accomplished by introducing a time delay between the formation of the ions and the application of the accelerating potential pulse.

See also delayed extraction.

From [346].

\section{5. time-of-flight mass spectrometer (TOF-MS)}

Mass spectrometer that separates ions by $\mathrm{m} / \mathrm{z}$ in a field-free region after acceleration through a fixed accelerating potential. Ions of the same initial translational energy and different $\mathrm{m} / \mathrm{z}$ require different times to traverse a given distance in the field-free region.

From $[5,6]$.

\section{6. tolyl ion}

Even-electron $\mathrm{C}_{7} \mathrm{H}_{7}{ }^{+}$ion with the same carbon skeleton as toluene that has lost a hydrogen atom from one of the ring carbons that carries the positive charge.

See also tropylium ion, benzyl ion.

From [57].

\section{7. top-down proteomics}

Method of protein identification that uses the $\mathrm{m} / \mathrm{z}$ selection of intact proteins followed by fragmentation and $\mathrm{m} / \mathrm{z}$ separation in a second stage of mass spectrometry. Mixtures of proteins must first be separated by liquid chromatography or other separation method prior to analysis by mass spectrometry.

From $[347,348]$.

\section{8. toroidal ion trap}

Ion trapping device based on the three-dimensional structure formed by rotating the cross-section of a quadrupole ion trap about the edge perpendicular to the plane of the ring electrode. The resulting trapping region is toroidal.

From [349].

\section{9. total ion chromatogram}

This term is deprecated.

See total ion current chromatogram (TICC). 


\section{0. total ion current (TIC)}

Obsolete: after mass analysis, before mass analysis

Sum of all the separate ion currents carried by the ions of different $\mathrm{m} / \mathrm{z}$ contributing to a complete mass spectrum or in a specified $\mathrm{m} / \mathrm{z}$ range of a mass spectrum.

From $[5,6]$.

\section{1. total ion current chromatogram (TICC)} reconstructed total ion current chromatogram

Deprecated: total ion chromatogram.

Chromatogram created by plotting the total ion current in a series of mass spectra recorded as a function of retention time.

From [350].

\section{2. total ion current electropherogram} total ion current electrophorogram

Plot of the total ion current in each of a series of mass spectra that are recorded as a function of electrophoresis time.

From [351].

\section{3. total ion current electrophorogram}

See total ion current electropherogram.

\section{4. total ion current profile}

Any plot of total ion current in a series of mass spectra recorded as a function of time, including total ion current chromatogram, total ion current electropherogram, flow injection mass spectrometry, or any other time dependent sampling.

See also extracted ion profile.

From [352].

\section{5. transmission efficiency}

Ratio of the number of ions leaving a region of a mass spectrometer to the number entering that region. From $[5,6]$.

\section{6. transmission quadrupole mass spectrometer quadrupole mass analyzer quadrupole mass filter quadrupole mass spectrometer}

Mass spectrometer that consists of an array of four parallel rod electrodes whose centers form the corners of a square and whose opposing electrode pairs are connected. The electric potential applied between the connected pairs of rods is a superposition of a static potential and a sinusoidal radio frequency potential. The motion of an ion in the $x-y$ plane perpendicular to the length direction of the rod 
electrodes is described by the Mathieu equation whose solutions show that ions in a particular $\mathrm{m} / \mathrm{z}$, range can be transmitted along the $z$-axis.

Revised from [5,6] using additional information from [353].

\section{7. transport region}

Region in a mass spectrometer in which an ion beam is transported from the ion source to the ion optics or from one ion optical element to the next.

From [354].

\section{8. triple quadrupole mass spectrometer}

Tandem mass spectrometer comprising two transmission quadrupole mass spectrometers in series, with a (non-selecting) RF-only quadrupole (or other multipole) between them to act as a collision cell. From [78].

\section{9. tropylium ion}

Delocalized carbenium ion, cycloheptatrienylium, $\mathrm{C}_{7} \mathrm{H}_{7}{ }^{+}$, derived formally by detachment of one hydride ion from the $\mathrm{CH}_{2}$ group of cyclohepta-1,3,5-triene and substitution derivatives thereof.

See also benzyl ion, tolyl ion.

From [6].

540. unified atomic mass unit, $u$ dalton

Non-SI unit of mass defined as one-twelfth of the mass of one atom of ${ }^{12} \mathrm{C}$ at rest in its ground state and equal to $1.660538921(73) \times 10^{-27} \mathrm{~kg}$ where the digits in parentheses indicate the estimated uncertainty in the final two digits of the value. Equivalent to the dalton (Da) unit.

Note: $\quad$ The abbreviation amu for atomic mass unit is deprecated; it has been used to denote atomic masses measured relative to a single atom of ${ }^{16} \mathrm{O}$, or to the isotope-averaged mass of an oxygen atom, or to a single atom of ${ }^{12} \mathrm{C}$.

Revised from [6] using additional information from [46].

\section{1. unimolecular dissociation}

Fragmentation reaction in which the molecularity of the reaction system is unity. The dissociation may arise from the extra energy acquired by a metastable ion produced in the ion source or that provided by collisional excitation of a stable ion.

From [355].

\section{2. unit mass resolution}

Mass resolution such that it is possible to clearly distinguish a peak corresponding to a singly charged ion from its neighbors $1 \mathrm{u}$ away, usually with no more than 5-10\% overlap.

Note: This term is most frequently used to describe resolution obtained using a Paul ion trap or a transmission quadrupole mass spectrometer.

From [356]. 


\section{3. unstable ion}

Nascent ion that is either inherently unstable or formed with sufficient energy to dissociate within the region of formation such as the ion source or collision cell.

Revised from $[5,6]$.

\section{4. vertical ionization}

Process in which one or more electrons are removed from or added to a molecule without a change in the positions of the atoms.

Note 1: The resulting ion is typically in an excited vibrational state.

Note 2: Such a "frozen nuclei" process is called a Franck-Condon process in optical specFrom $[5,6]$. troscopy.

\section{5. v-ion}

High-energy product ion of a protonated peptide formally equivalent to a y-ion with the ionizing proton on the $\mathrm{N}$-terminus, but with elimination of $\mathrm{HR}$ from the $\mathrm{N}$-terminus where $\mathrm{R}$ is the side-chain on the $\mathrm{C}$-atom of the $\mathrm{N}$-terminal residue, to give a $\left(\mathrm{H}_{2} \mathrm{~N}^{+}=\mathrm{CH}-\mathrm{CO}--\right)$ structure.

From [112].

\section{6. volumetric internal standard}

Compound added in a fixed reproducible amount to a solution to be analyzed by gas chromatography to permit corrections for uncontrolled variations in injected volumes.

See also surrogate internal standard.

From [357].

\section{Wahrhaftig diagram}

Diagram illustrating the relative contributions in unimolecular dissociation of an ion, for example, by homolytic cleavage of a single bond, and fragmentation following rearrangement. The diagram is in two parts with a common horizontal axis representing the internal energy of the ion. The vertical axis in one part of the diagram shows the two types of rate constant on a logarithmic scale, and that for the other portion of the diagram indicates the distribution function for internal energy of the ion.

From [24,358].

\section{Wien filter}

Mass spectrometer in which $\mathrm{m} / \mathrm{z}$ separation is achieved using crossed electric and magnetic fields.

Note: It can also be operated as a charged particle energy analyzer or monochromator.

From [359].

\section{9. w-ion}

High-energy product ion of a protonated peptide formally equivalent to a $z$-ion that has lost the part of the $\mathrm{N}$-terminal side-chain starting at the $\gamma$-carbon (if any).

From [112]. 
550. $x$-ion

Fragment ion containing the peptide C-terminus formed upon dissociation of a peptide ion at the peptide backbone $\mathrm{C}-\mathrm{C}$ bond.

From [28].

551. $y$-ion

Fragment ion containing the peptide $\mathrm{C}$-terminus formed upon dissociation of a peptide ion at the peptide backbone $\mathrm{C}-\mathrm{N}$ bond.

From [28].

552. z-ion

Fragment ion containing the peptide C-terminus formed upon dissociation of a peptide ion at the peptide backbone $\mathrm{N}-\mathrm{C}$ bond.

From [28].

\section{MEMBERSHIP OF SPONSORING BODY}

Membership of the Analytical Chemistry Division Committee during the preparation of this report (2003-2006) was as follows:

President: R. Lobinski (France); Titular Members: M. L. Bonardi (Italy); P. De Bièvre (Belgium); A. Fajgelj (Slovenia); D. B. Hibbert (Australia); J. Å. Jönsson (Sweden); J. Labuda (Slovakia); W. Lund (Norway); H. K. J. Powell (New Zealand); R. M. Smith (UK); Associate Members: Z. Chai (China/Beijing); H. Gamsjäger (Austria); U. Karst (Netherlands); W. Kutner (Poland); P. Minkkinen (Finland); K. K. Murray (USA); National Representatives: E. A. G. Zagatto (Brazil); C. Balarew (Bulgaria); Z. Mester (Canada); W. Wang (China); H. Watarai (Japan); B. Spivakov (Russia); E. Dominguez (Spain); S. Kocaoba (Turkey); Provisional Member: N. Torto (Botswana).

\section{REFERENCES}

1. J. F. J. Todd. Pure Appl. Chem. 63, 1541 (1991).

2. J. F. J. Todd. Int. J. Mass Spectrom. Ion Processes 142, 211 (1995).

3. K. Biemann. Pure Appl. Chem. 65, 1021 (1993).

4. Y.-F. Liu, Z.-Y. Guo, X.-Q. Liu, T. Qu, J.-L. Xie. Pure Appl. Chem. 66, 305 (1994).

5. IUPAC. Compendium of Analytical Nomenclature, $3^{\text {rd }}$ ed. (the "Orange Book"). Prepared for publication by J. Inczédy, T. Lengyel, A. M. Ure, Blackwell Science, Oxford (1998).

6. IUPAC. Compendium of Chemical Terminology, $2^{\text {nd }}$ ed. (the "Gold Book"). Compiled by A. D. McNaught and A. Wilkinson. Blackwell Scientific Publications, Oxford (1997). XML on-line corrected version: http://dx.doi.org/10.1351/goldbook (2006-) created by M. Nic, J. Jirat, B. Kosata; updates compiled by A. Jenkins.

7. IUPAC. Quantities, Units and Symbols in Physical Chemistry, $3^{\text {rd }}$ ed. (the "Green Book"). Prepared for publication by E. R. Cohen, T. Cvitaš, J. G. Frey, B. Holmström, K. Kuchitsu, R. Marquardt, I. Mills, F. Pavese, M. Quack, J. Stohner, H. L. Strauss, M. Takami, A. J. Thor, RSC Publishing, Cambridge, UK (2007).

8. P. Price. J. Am. Soc. Mass Spectrom. 2, 336 (1991).

9. American Society for Mass Spectrometry: http://www.asms.org/.

10. O. D. Sparkman. Mass Spec Desk Reference, Global View Publishing, Pittsburgh (2006).

11. A. I. Mallet, S. Down. Dictionary of Mass Spectrometry, John Wiley, Chichester (2010). 
12. K. K. Murray. J. Chromatogr., A 1217, 3922 (2010).

13. R. G. Cooks, A. L. Rockwood. Rapid Commun. Mass Spectrom. 5, 93 (1991).

14. J. Beynon. Pure Appl. Chem. 50, 65 (1978).

15. A. G. Marshall, C. L. Hendrickson, G. S. Jackson. Mass Spectrom. Rev. 17, 1 (1998).

16. M. Bursey. Mass Spectrom. Rev. 10, 1 (1991).

17. J. Adams. J. Am. Soc. Mass Spectrom. 3, 473 (1992).

18. J. Lederberg, M. Wightman. Anal. Chem. 36, 2362 (1964).

19. F. W. Karasek, R. J. Smythe. Anal. Chem. 43, 2008 (1971).

20. T. Hirschfeld. Anal. Chem. 52, 297A (1980).

21. Conventions adopted by RCM in Advice to Authors. Rapid Commun. Mass Spectrom. 17, Issue 1 (2003).

22. S. A. Gerber, J. Rush, O. Stemman, M. W. Kirschner, S. P. Gygi. Proc. Natl. Acad. Sci. USA 100, $6940(2003)$.

23. F. White, T. Collins. Appl. Spectrosc. 8, 169 (1954).

24. F. W. McLafferty, F. Turecek. Interpretation of Mass Spectra, University Science Books, Mill Valley, CA (1993).

25. A. W. T. Bristow, K. S. Webb. J. Am. Soc. Mass Spectrom. 14, 1086 (2003).

26. R. D. Smith, J. A. Loo, C. G. Edmonds, C. J. Barinaga, H. R. Udseth. Anal. Chem. 62, 882 (1990).

27. T. P. Conrads, G. A. Anderson, T. D. Veenstra, L. Paša-Tolić, R. D. Smith. Anal. Chem. 72, 3349 (2000).

28. P. Roepstorff, J. Fohlman. Biomed. Mass Spectrom. 11, 601 (1984).

29. R. Houriet, T. Gäumann. Helvetica Chim. Acta 59, 119 (1976).

30. E. Kluft, N. M. M. Nibbering. Int. J. Mass Spectrom. Ion Processes 92, 171 (1989).

31. H. E. Lumpkin, G. E. Taylor. Anal. Chem. 33, 476 (1961).

32. R. Houriet, T. A. Elwood, J. H. Futrell. J. Am. Chem. Soc. 100, 2320 (1978).

33. N. S. Shuman, W. R. Stevens, K. Lower, T. Baer. J. Phys. Chem. A 113, 10710 (2009).

34. R. G. Cooks, Z. Ouyang, Z. Takats, J. M. Wiseman. Science 311, 1566 (2006).

35. T. M. Annesley. Clin. Chem. 49, 1041 (2003).

36. B. Gulbakan, E. Yasun, M. I. Shukoor, Z. Zhu, M. You, X. Tan, H. Sanchez, D. H. Powell, H. Dai, W. Tan. J. Am. Chem. Soc. 132, 17408 (2010).

37. P. H. Hemberger, J. A. Laramée, A. R. Hubik, R. G. Cooks. J. Phys. Chem. 85, 2335 (1981).

38. L. Van Vaeck, H. Struyf, W. V. Roy, F. Adams. Mass Spectrom. Rev. 13, 209 (1994).

39. J. A. Hill, S. A. Martin, J. E. Biller, K. Biemann. Biomed. Env. Mass Spectrom. 17, 147 (1988).

40. L. C. Taylor, D. A. Brent, J. S. Cottrell. Biochem. Biophys. Res. Commun. 145, 542 (1987).

41. D. K. Bohme, D. B. Dunkin, F. C. Fehsenfeld, E. E. Ferguson. J. Chem. Phys. 51, 863 (1969).

42. D. I. Carroll, I. Dzidic, R. N. Stillwell, K. D. Haegele, E. C. Horning. Anal. Chem. 47, 2369 (1975).

43. E. Rosenberg. J. Chromatogr., A 1000, 841 (2003).

44. V. V. Laiko, M. A. Baldwin, A. L. Burlingame. Anal. Chem. 72, 652 (2000).

45. D. B. Robb, T. R. Covey, A. P. Bruins. Anal. Chem. 72, 3653 (2000).

46. P. J. Mohr, B. N. Taylor, D. B. Newell. Rev. Mod. Phys. 84, 1527 (2012).

47. B. H. Bransden, A. Dalgarno. Proc. Phys. Soc., London A 69, 65 (1956).

48. S. Singh, R. K. Boyd, F. M. Harris, J. H. Beynon. Proc. R. Soc. London, Ser. A 373 (1985).

49. I. Manisali, D. Chen, B. Schneider. Trends Anal. Chem. 25, 243 (2006).

50. C. Pan, G. Zhu, R. F. Browner. J. Anal. At. Spectrom. 7, 1231 (1992).

51. J. Yergey, D. Heller, G. Hansen, R. J. Cotter, C. Fenselau. Anal. Chem. 55, 353 (1983).

52. R. E. March. J. Mass Spectrom. 32, 351 (1997).

53. J. A. Marto, S. Guan, A. G. Marshall. Rapid Commun. Mass Spectrom. 8, 615 (1994).

54. C. L. Hendrickson, D. A. Laude. Anal. Chem. 67, 1717 (1995). 
55. W. M. A. Niessen. In The Encyclopedia of Mass Spectrometry, M. L. Gross, R. M. Caprioli (Eds.), Elsevier (2006).

56. T. P. J. Izod, J. M. Tedder. Proc. R. Soc. London, Ser. A 337, 333 (1974).

57. T. Baer, J. C. Morrow, J. D. Shao, S. Olesik. J. Am. Chem. Soc. 110, 5633 (1988).

58. R. C. Dunbar. Mass Spectrom. Rev. 23, 127 (2004).

59. N. L. Kelleher, H. Y. Lin, G. A. Valaskovic, D. J. Aaserud, E. K. Fridriksson, F. W. McLafferty. J. Am. Chem. Soc. 121, 806 (1999).

60. B. T. Chait. Science 314, 65 (2006).

61. D. J. Douglas. Mass Spectrom. Rev. 28, 937 (2009).

62. J. F. J. Todd. Mass Spectrom. Rev. 10, 3 (1991).

63. J. Meija, J. A. Caruso. J. Am. Soc. Mass Spectrom. 15, 654 (2004).

64. C. J. Hogan, J. A. Carroll, H. W. Rohrs, P. Biswas, M. L. Gross. Anal. Chem. 81, 369 (2009).

65. B. Gologan, J. R. Green, J. Alvarez, J. Laskin, R. G. Cooks. Phys. Chem. Chem. Phys. 7, 1490 (2005).

66. C. Cheng, D. Giblin, M. L. Gross. J. Am. Soc. Mass Spectrom. 9, 216 (1998).

67. A. Savagnac, H. Aurelle, C. Casas, F. Couderc, P. Gavard, D. Prome, J. C. Prome. Chem. Phys. Lipids 51, 31 (1989).

68. K. B. Tomer, N. J. Jensen, M. L. Gross, J. Whitney. Biomed. Environ. Mass Spectrom. 13, 265 (1986).

69. X. Czeszak, W. Morelle, G. Ricart, D. Tétaert, J. Lemoine. Anal. Chem. 76, 4320 (2004).

70. M. Rabrenović, C. J. Proctor, T. Ast, C. G. Herbert, A. G. Brenton, J. H. Beynon. J. Phys. Chem. 87, 3305 (1983).

71. P. S. Rudolph, C. E. Melton. J. Chem. Phys. 32, 586 (1960).

72. J. M. Dyke, A. M. Shaw, T. G. Wright. J. Phys. Chem. 98, 6327 (1994).

73. T. J. Cornish, R. J. Cotter. Rapid. Commun. Mass Spectrom. 8, 781 (1994).

74. B. L. Schram, B. Adamczyks, A. J. H. Boerboom. J. Sci. Instrum. 43, 638 (1966).

75. J. M. Miller, J. Ross, J. Rustenburg, G. L. Wilson. Anal. Chem. 45, 627 (1973).

76. D. J. Douglas, J. B. French. J. Am. Soc. Mass Spectrom. 3, 398 (1992).

77. R. K. Boyd, P. A. Bott, B. R. Beer, D. J. Harvan, J. R. Hass. Anal. Chem. 59, 189 (1987).

78. A. K. Shukla, J. H. Futrell. Mass Spectrom. Rev. 12, 211 (1993).

79. S. A. McLuckey, G. L. Glish, R. G. Cooks. Int. J. Mass Spectrom. Ion Phys. 39, 219 (1981).

80. R. A. Yost, C. G. Enke. J. Am. Chem. Soc. 100, 2274 (1978).

81. S. D. Tanner, V. I. Baranov, D. R. Bandura. Spectrochim. Acta, Part B 57, 1361 (2002).

82. A. P. Bruins, T. R. Covey, J. D. Henion. Anal. Chem. 59, 2642 (1987).

83. K. B. Tomer, C. R. Guenat, L. J. Deterding. Anal. Chem. 60, 2232 (1988).

84. S. S. Brenner, J. T. McKinney. Rev. Sci. Instrum. 43, 1264 (1972).

85. R. M. Caprioli. Trends Anal. Chem. 7, 328 (1988).

86. R. M. Caprioli, T. Fan, J. S. Cottrell. Anal. Chem. 58, 2949 (1986).

87. L. Li, A. P. L. Wang, L. D. Coulson. Anal. Chem. 65, 493 (1993).

88. R. J. Beuhler, L. Friedman. Int. J. Mass Spectrom. Ion Phys. 23, 81 (1977).

89. G. C. Stafford. Environ. Health Perspect. 36, 85 (1980).

90. K. Rinn, A. Muller, H. Eichenauer, E. Salzborn. Rev. Sci. Instrum. 53, 829 (1982).

91. F. W. Peek. Dielectric Phenomena in High-Voltage Engineering, McGraw-Hill (1929).

92. E. C. Huang, T. Wachs, J. J. Conboy, J. D. Henion. Anal. Chem. 62, 713 (1990).

93. C. M. Whitehouse, R. N. Dreyer, M. Yamashita, J. B. Fenn. Anal. Chem. 57, 675 (1985).

94. B. Thomson, J. Iribarne, P. Dziedzic. Anal. Chem. 54, 2219 (1982).

95. W. M. A. Niessen, A. P. Tinke. J. Chromatogr., A 703, 37 (1995).

96. R. C. Willoughby, R. F. Browner. Anal. Chem. 56, 2626 (1984).

97. M. M. Cordero, T. J. Cornish, R. J. Cotter, I. A. Lys. Rapid Commun. Mass Spectrom. 9, 1356 (1995). 
98. W. K. Van Asselt, B. Poelsema, A. L. Boers. J. Phys. D: Appl. Phys. 11, L107 (1978).

99. M. Mann, R. C. Hendrickson, A. Pandey. Ann. Rev. Biochem. 70, 437 (2001).

100. H. S. Gadgil, G. D. Pipes, T. M. Dillon, M. J. Treuheit, P. V. Bondarenko. J. Am. Soc. Mass Spectrom. 17, 867 (2006).

101. B. N. Colby. J. Am. Soc. Mass Spectrom. 3, 558 (1992).

102. R. J. Cotter. Time-of-Flight Mass Spectrometry: Instrumentation and Applications in Biological Research, American Chemical Society, Washington, DC (1997).

103. M. L. Vestal, P. Juhasz, S. A. Martin. Rapid Commun. Mass Spectrom. 9, 1044 (1995).

104. M. Takayama. Rapid Commun. Mass Spectrom. 8, 309 (1994).

105. M. Vincenti. Int. J. Mass Spectrom. 212, 505 (2001).

106. U. Schade, R. Stoll, F. W. Röllgen. Org. Mass Spectrom. 16, 441 (1981).

107. Z. Takats, I. Cotte-Rodriguez, N. Talaty, H. Chen, R. G. Cooks. Chem. Commun. 1950 (2005).

108. K. L. Busch, S. E. Unger, A. Vincze, R. G. Cooks, T. Keough. J. Am. Chem. Soc. 104, 1507 (1982).

109. J. Wei, J. M. Buriak, G. Siuzdak. Nature 399, 243 (1999).

110. S. A. Carr, M. J. Huddleston, M. F. Bean. Protein Sci. 2, 183 (1993).

111. H. Hayen, A. Michels, J. Franzke. Anal. Chem. 81, 10239 (2009).

112. R. S. Johnson, S. A. Martin, K. Biemann. Int. J. Mass Spectrom. Ion Processes 86, 137 (1988).

113. R. J. Cotter. Anal. Chem. 51, 317 (1979).

114. E. Gelpí. J. Chromatogr., A 1000, 567 (2003).

115. A. E. Ashcroft. Ionization Methods in Organic Mass Spectrometry, Royal Society of Chemistry (1997).

116. J. D. Henion, G. A. Maylin. Biomed. Mass Spectrom. 7, 115 (1980).

117. W. Sandner, R. Kachru, K. A. Safinya, F. Gounand, W. E. Cooke, T. F. Gallagher. Phys. Rev. A 27, 1717 (1983).

118. L. Song, A. D. Wellman, H. Yao, J. E. Bartmess. J. Am. Soc. Mass Spectrom. 18, 1789 (2007).

119. T. M. Sack, R. L. Cerny, M. L. Gross. J. Am. Chem. Soc. 107, 4562 (1985).

120. S. Hammerum. Mass Spectrom. Rev. 7, 123 (1988).

121. P. C. Price, H. S. Swofford, S. E. Buttrill. Anal. Chem. 49, 1487 (1977).

122. S. F. Wong, C. K. Meng, J. B. Fenn. J. Phys. Chem. 92, 546 (1988).

123. B. M. Kohli, J. K. Eng, R. M. Nitsch, U. Konietzko. Rapid Commun. Mass Spectrom. 19, 589 (2005).

124. H. A. Donsig, J. C. Vickerman. J. Chem. Soc., Faraday Trans. 93, 2755 (1997).

125. A. Adams, F. H. Read. J. Phys. E 5, 150 (1972).

126. B. N. Colby, C. A. Evans. Anal. Chem. 45, 1884 (1972).

127. C. A. Evans, C. D. Hendricks. Rev. Sci. Instrum. 43, 1527 (1972).

128. D. S. Simons, B. N. Colby, J. C. A. Evans. Int. J. Mass Spectrom. Ion Phys. 15, 291 (1974).

129. R. A. Zubarev, N. L. Kelleher, F. W. McLafferty. J. Am. Chem. Soc. 120, 3265 (1998).

130. H. K. Lim, D. Andrenyak, P. Francom, R. L. Foltz, R. T. Jones. Anal. Chem. 60, 1420 (1988).

131. J. E. Syka, J. J. Coon, M. J. Schroeder, J. Shabanowitz, D. F. Hunt. Proc. Natl. Acad. Sci. USA 101, 9528 (2004).

132. J. B. Fenn, M. Mann, C. K. Meng, S. F. Wong, C. M. Whitehouse. Science 246, 64 (1989).

133. M. Dole, L. L. Mack, R. L. Hines, R. C. Mobley, L. D. Ferguson, M. B. Alice. J. Chem. Phys. 49, 2240 (1968).

134. J. H. Moore, C. C. Davis, M. A. Coplan. Building Scientific Apparatus, Westview Press (2002).

135. M. Karni, A. Mandelbaum. Org. Mass Spectrom. 15, 53 (1980).

136. S. Kim, R. P. Rodgers, A. G. Marshall. Int. J. Mass Spectrom. 251, 260 (2006).

137. J. J. Vrbanac, W. E. Braselton, J. F. Holland, C. C. Sweeley. J. Chromatogr. 239, 265 (1982).

138. V. T. Vu, F. P. Abramson. Biomed. Mass Spectrom. 5, 686 (1978).

139. M. Pelzing, C. Neususs. Electrophoresis 26, 2717 (2005).

(C) 2013, IUPAC

Pure Appl. Chem., Vol. 85, No. 7, pp. 1515-1609, 2013 
140. X. Zhao, Y. Lu, D. R. Phillips, H.-M. Hwang, I. R. Hardin. J. Chromatogr, A 1159, 217 (2007).

141. P. Mauri, M. Minoggio, P. Simonetti, C. Gardana, P. Pietta. Rapid Commun. Mass Spectrom. 16, 743 (2002).

142. H. R. Morris, M. Panico, M. Barber, R. S. Bordoli, R. D. Sedgwick, A. N. Tyler. Biochem. Biophys. Res. Comm. 101, 623 (1981).

143. M. Hamdan. Org. Mass Spectrom. 27, 759 (1992).

144. R. A. Klein. J. Lipid Res. 12, 628 (1971).

145. P. H. Dawson. Quadrupole Mass Spectrometry and Its Applications, Elsevier, New York (1976).

146. L. O. G. Weidolf, E. D. Lee, J. D. Henion. Biol. Mass Spectrom. 15, 283 (1988).

147. H. F. Schröder. J. Chromatogr., A 647, 219 (1993).

148. J. A. Hill, J. E. Biller, S. A. Martin, K. Biemann, K. Yoshidome, K. Sato. Int. J. Mass Spectrom. Ion Processes 92, 211 (1989).

149. M. J. Farncombe, R. S. Mason, K. R. Jennings, J. Scrivens. Int J. Mass Spectrom. Ion Phys. 44, 91 (1982).

150. D. H. Smith, W. J. Yeager, T. C. Rindfleisch. Anal. Chem. 50, 1585 (1978).

151. M. Scigelova, M. Hornshaw, A. Giannakopulos, A. Makarov. Mol. Cell. Proteomics 10, 1 (2011).

152. G. Glish. J. Am. Soc. Mass Spectrom. 2, 349 (1991).

153. G. C. Thorne, K. D. Ballard, S. J. Gaskell. J. Am. Soc. Mass Spectrom. 1, 249 (1990).

154. W. M. Brubaker. Adv. Mass Spectrom. 4, 293 (1968).

155. R. S. Gohlke, F. W. McLafferty. J. Am. Soc. Mass Spectrom. 4, 367 (1993).

156. J. Coburn, W. Harrison. Appl. Spectrosc. Rev. 17, 95 (1981).

157. F. L. King, J. Teng, R. E. Steiner. J. Mass Spectrom. 30, 1061 (1995).

158. R. P. Schmid, C. Weickhardt. Int. J. Mass Spectrom. 206, 181 (2001).

159. D. L. Bricker, T. A. Adams, D. H. Russell. Anal. Chem. 55, 2417 (1983).

160. N. C. Blais, D. G. Truhlar. Astrophys. J. 258, L79 (1982).

161. R. Purves, R. Guevremont, S. Day, C. Pipich, M. Matyjaszczyk. Rev. Sci. Instrum. 69, 4094 (1998).

162. G. E. Spangler. Int. J. Ion Mobility Spectrom. 4, 71 (2001).

163. W. Blum, E. Schlumpf, J. G. Liehr, W. J. Richter. Tetrahedron Lett. 17, 565 (1976).

164. S. Sethi, D. Smith, J. McCloskey. Biochem. Biophys. Res. Commun. 112, 126 (1983).

165. R. B. Woodward, R. Hoffmann. The Conservation of Orbital Symmetry, Verlag Chemie Academic Press (2004).

166. J. van der Greef, W. M. A. Niessen. Int. J. Mass Spectrom. Ion Proc. 118/119, 857 (1992).

167. M. Kamruddin, P. K. Ajikumar, S. Dash, A. K. Tyagi, B. Raj. Bull. Mater. Sci. 26, 449 (2003).

168. M. B. Comisarow. J. Chem. Phys. 69, 4097 (1978).

169. S. D. Fuerstenau, W. H. Benner. Rapid Commun. Mass Spectrom. 9, 1528 (1995).

170. D. Kingham, A. Bayly, D. Fathers, P. Vohralik, J. Walls, A. Waugh. Scan. Microsc. 1, 463 (1987).

171. R. S. Houk, V. A. Fassel, G. D. Flesch, H. J. Svec, A. L. Gray, C. E. Taylor. Anal. Chem. 52, 2283 (1980).

172. H. E. Taylor. Inductively Coupled Plasma-Mass Spectrometry, Academic Press, Boston (2001).

173. D. S. Bomse, D. W. Berman, J. Beauchamp. J. Am. Chem. Soc. 103, 3967 (1981).

174. C. H. Watson, G. Baykut, M. A. Battiste, J. R. Eyler. Anal. Chim. Acta 178, 125 (1985).

175. G. Baykut, C. H. Watson, R. R. Weller, J. R. Eyler. J. Am. Chem. Soc. 107, 8036 (1985).

176. J. Josephs. Rapid Commun. Mass Spectrom. 9, 1270 (1995).

177. S. Sekiya, Y. Wada, K. Tanaka. Anal. Chem. 76, 5894 (2004).

178. C. R. Mallet, Z. Lu, J. R. Mazzeo. Rapid Commun. Mass Spectrom. 18, 49 (2004).

179. J. V. Iribarne, B. A. Thomson. J. Chem. Phys. 64, 2287 (1976).

180. V. Znamenskiy, I. Marginean, A. Vertes. J. Phys. Chem. A 107, 7406 (2003).

181. S. A. Shaffer, K. Q. Tang, G. A. Anderson, D. C. Prior, H. R. Udseth, R. D. Smith. Rapid Commun. Mass Spectrom. 11, 1813 (1997). 
182. A. B. Kanu, P. Dwivedi, M. Tam, L. Matz, H. H. Hill. J. Mass Spectrom. 43, 1 (2008).

183. S. A. McLuckey, J. L. Stephenson Jr. Mass Spectrom. Rev. 17, 369 (1998).

184. K. Dreisewerd. Chem. Rev. 103, 395 (2003).

185. J. N. Brownlee. J. Atmos. Terr. Phys. 37, 1139 (1975).

186. G. E. Spangler, J. P. Carrico. Int. J. Mass Spectrom. Ion Phys. 52, 267 (1983).

187. R. D. Bowen. Acc. Chem. Res. 24, 364, (1991).

188. T. Matsuo, M. Toyoda, T. Sakurai, M. Ishihara. J. Mass Spectrom. 32, 1179 (1997).

189. E. de Hoffmann, J. Charette, V. Stroobant, J. Trottier. Mass Spectrometry: Principles and Applications, John Wiley, Chichester (1996).

190. F. Dubois, R. Knochenmuss, R. Zenobi. Int. J. Mass Spectrom. Ion Processes 169, 89 (1997).

191. J. P. Holder, L. Gruber, H. E. DeWitt, B. R. Beck, D. A. Church, D. Schneider. Phys. Scr., T 92 , 158 (2001).

192. P. L. Ross, Y. N. Huang, J. N. Marchese, B. Williamson, K. Parker, S. Hattan, N. Khainovski, S. Pillai, S. Dey, S. Daniels, S. Purkayastha, P. Juhasz, S. Martin, M. Bartlet-Jones, F. He, A. Jacobson, D. J. Pappin. Mol. Cell Proteomics 3, 1154 (2004).

193. L. R. Zieske. J. Exp. Bot. 57, 1501 (2006).

194. S. P. Gygi, B. Rist, S. A. Gerber, F. Turecek, M. H. Gelb, R. Aebersold. Nat. Biotech. 17, 994 (1999).

195. L. J. Moore, L. A. Machlan. Anal. Chem. 44, 2291 (1972).

196. T. B. Coplen. Rapid Commun. Mass Spectrom. 25, 2538 (2011).

197. W. A. Brand, S. S. Assonov, T. B. Coplen. Pure Appl. Chem. 82, 1719 (2010).

198. W. A. Brand. J. Mass Spectrom. 31, 225 (1996).

199. R. Ryhage. Anal. Chem. 36, 759 (1964).

200. G. A. Junk. Int. J. Mass Spectrom. Ion Phys. 8, 1 (1972).

201. J. Laskin, C. Lifshitz. J. Mass Spectrom. 36, 459 (2001).

202. C. Lifshitz. Eur. J. Mass Spectrom. 8, 85 (2002).

203. A. Makarov. Anal. Chem. 72, 1156 (2000).

204. M. Bantscheff, M. Schirle, G. Sweetman, J. Rick, B. Kuster. Anal. Bioanal. Chem. 389, 1017 (2007).

205. R. E. Russo. Appl. Spectrosc. 49, 14A (1995).

206. J. M. Chen, C. C. Chang. J. Appl. Phys. 43, 3884 (1972).

207. E. D. Hardin, M. L. Vestal. Anal. Chem. 53, 1492 (1981).

208. E. Denoyer, R. Van Grieken, F. Adams, D. F. S. Natusch. Anal. Chem. 54, 26A (1982).

209. G. R. Janik, J. D. Prestage, L. Maleki. J. Appl. Phys. 67, 6050 (1990).

210. M. A. Baldwin, F. W. McLafferty. Org. Mass Spectrom. 7, 1111 (1973).

211. P. J. Arpino, B. G. Dawkins, F. W. McLafferty. J. Chromatogr. Sci. 12, 574 (1974).

212. N. J. Reinhoud, W. M. A. Niessen, U. R. Tjaden, L. G. Gramberg, E. R. Verheij, J. van der Greef. Rapid Commun. Mass Spectrom. 3, 348 (1989).

213. E. D. Lee, W. Muck, J. D. Henion, T. R. Covey. Biol. Mass Spectrom. 18, 253 (1989).

214. A. M. Falick, F. C. Walls, R. A. Laine. Anal. Biochem. 159, 132 (1986).

215. C. G. Edmonds, J. A. Loo, C. J. Barinaga, H. R. Udseth, R. D. Smith. J. Chromatogr., A 474, 21 (1989).

216. R. D. Smith, J. A. Olivares, N. T. Nguyen, H. R. Udseth. Anal. Chem. 60, 436 (2002).

217. K. B. Tomer. Chem. Rev. 101, 297 (2001).

218. J. V. Olsen, L. M. F. de Godoy, G. Li, B. Macek, P. Mortensen, R. Pesch, A. Makarov, O. Lange, S. Horning, M. Mann. Mol. Cell Proteomics 4, 2010 (2005).

219. A. Weisz, A. Mandelbaum, J. Shabanowitz, D. F. Hunt. Org. Mass Spectrom. 19, 238 (1984).

220. R. L. Hunter, M. G. Sherman, R. T. McIver. Int. J. Mass Spectrom. Ion Phys. 50, 259 (1983).

221. J. B. Jeffries, S. E. Barlow, G. H. Dunn. Int. J. Mass Spectrom. Ion Processes 54, 169 (1983).

222. J. H. Beynon, R. G. Cooks. Res. Dev. 22, 26 (1971).

(C) 2013, IUPAC

Pure Appl. Chem., Vol. 85, No. 7, pp. 1515-1609, 2013 
223. J. H. Beynon, S. Clough. J. Sci. Instrum. 35, 289 (1958).

224. E. Kendrick. Anal. Chem. 35, 2146 (1963).

225. K. V. Wood, A. H. Grange, J. W. Taylor. Anal. Chem. 50, 1652 (1978).

226. L.-S. Zheng, C. M. Karner, P. J. Brucat, S. H. Yang, C. L. Pettiette, M. J. Craycraft, R. E. Smalley. J. Chem. Phys. 85, 1681 (1986).

227. M. L. Gross, D. L. Rempel. Science 226, 261 (1984).

228. F. A. Londry, J. W. Hager. J. Am. Soc. Mass Spectrom. 14, 1130 (2003).

229. G. C. Stafford, P. E. Kelley, J. E. P. Syka, W. E. Reynolds, J. F. J. Todd. Int. J. Mass Spectrom. Ion Processes 60, 85 (1984).

230. F. P. Abramson. Anal. Chem. 47, 45 (1975).

231. P. H. Dawson, N. R. Whetten. Int. J. Mass Spectrom. Ion Phys. 2, 45 (1969).

232. M. Karas, D. Bachman, U. Bahr, F. Hillenkamp. Int. J. Mass Spectrom. Ion Processes 78, 53 (1987).

233. A. Cappiello, G. Famiglini, P. Palma, E. Pierini, V. Termopoli, H. Trufelli. Anal. Chem. 80, 9343 (2008).

234. R. C. Johnson, R. G. Cooks, T. M. Allen, M. E. Cisper, P. H. Hemberger. Mass Spectrom. Rev. 19, 1 (2000).

235. V. I. Dukhanov, A. G. Zelenkov, A. A. Kurashov, I. B. Mazurov, Y. F. Rodionov, I. N. Serikov, V. P. Tarasevich. J. Radioanal. Chem. 58, 161 (1980).

236. M. R. Emmett, P. E. Andren, R. M. Caprioli. J. Neurosci. Meth. 62, 141 (1995).

237. D. R. Olander, W. W. Waddel. Anal. Chem. 40, 1687 (1968).

238. W. Fock. Anal. Chem. 47, 2447 (2002).

239. P. Arpino. Fresenius' J. Anal. Chem. 337, 667 (1990).

240. P. O. Edlund, J. D. Henion. J. Chromatogr. Sci. 27, 274 (1989).

241. W. H. McFadden, H. L. Schwarz, S. Evans. J. Chromatogr., A 122, 389 (1976).

242. D. Zakett, A. Schoen, R. Cooks, P. Hemberger. J. Am. Chem. Soc. 103, 1295 (1981).

243. G. L. Glish. Analyst 119, 533 (1994).

244. C. B. Douthitt. Anal. Bioanal. Chem. 390, 437 (2008).

245. D. A. Wolters, M. P. Washburn, J. R. Yates. Anal. Chem. 73, 5683 (2001).

246. D. Zakett, R. G. A. Flynn, R. G. Cooks. J. Phys. Chem. 82, 2359 (1977).

247. M. Wilm, M. Mann. Anal. Chem. 68, 1 (1996).

248. F. W. McLafferty. Science 247, 925 (1990).

249. D. Zakett, A. E. Schoen, R. W. Kondrat, R. G. Cooks. J. Am. Chem. Soc. 101, 6781 (1979).

250. W. H. Johnson, A. O. Nier. Phys. Rev. 105, 1014 (1957).

251. M. W. Senko, S. C. Beu, F. W. McLafferty. Anal. Chem. 66, 415 (1994).

252. J. A. Loo, H. R. Udseth, R. D. Smith. Rapid Commun. Mass Spectrom. 2, 207 (1988).

253. M. Yamashita, J. B. Fenn. J. Phys. Chem. 88, 4451 (1984).

254. W. M. A. Niessen. J. Chromatogr., A 856, 179 (1999).

255. F. L. Wiseman, F. Ozturk, M. C. Zerner, J. R. Eyler. Int. J. Chem. Kin. 22, 1189 (1990).

256. C. Blakley, J. Carmody, M. Vestal. Clin. Chem. 26, 1467 (1980).

257. A. Cappiello. Mass Spectrom. Rev. 15, 283 (1996).

258. C. G. Hammar, G. Pettersson, P. T. Carpenter. Biomed. Mass Spectrom. 1, 397 (1974).

259. F. Li, J. Maguigad, M. Pelzer, X. Jiang, Q. C. Ji. Rapid Commun. Mass Spectrom. 22, 486 (2008).

260. P. Z. Vroon, B. van der Wagt, J. M. Koornneef, G. R. Davies. Anal. Bioanal. Chem. 390, 465 (2008).

261. K. P. Wanczek. Int. J. Mass Spectrom. Ion Processes 95, 1 (1989).

262. D. J. C. Pappin, P. Hojrup, A. J. Bleasby. Curr. Biol. 3, 327 (1993).

263. M. Mann, M. Wilm. Anal. Chem. 66, 4390 (1994).

264. I. W. Griffiths, E. S. Mukhtar, R. E. March, F. M. Harris, J. H. Beynon. Int. J. Mass Spectrom. Ion Phys. 39, 125 (1981). 
265. T. R. Covey, E. C. Huang, J. D. Henion. Anal. Chem. 63, 1193 (1991).

266. A. M. Falick, K. F. Medzihradszky, F. C. Walls. Rapid Commun. Mass Spectrom. 4, 318 (1990).

267. G. H. Wang, W. Aberth, A. M. Falick. Int. J. Mass. Spectrom. Ion Processes 69, 233 (1986).

268. B. Spengler, D. Kirsch, R. Kaufmann. J. Phys. Chem. 96, 9678 (1992).

269. F. W. McLafferty, R. H. Hertel, R. D. Villwock. Org. Mass Spectrom. 9, 690 (1974).

270. C. Sweeley, W. Elliott, I. Fries, R. Ryhage. Anal. Chem. 38, 1549 (1966).

271. S. A. Martin, K. Biemann. Int. J. Mass Spectrom. Ion Processes 78, 213 (1987).

272. J. W. Larson, R. L. Clair, T. B. McMahon. Can. J. Chem. 60, 542 (1982).

273. R. G. Ewing, G. A. Eiceman, C. S. Harden, J. A. Stone. Int. J. Mass Spectrom. 255-256, 76 (2006).

274. I. Lüderwald. Pure Appl. Chem. 54, 255 (1982).

275. A. W. Colburn, A. E. Giannakopulos, P. J. Derrick, M. Von Raumer. Eur. J. Mass Spectrom. 6, $523(2000)$.

276. J. P. Speir, G. S. Gorman, C. C. Pitsenberger, C. A. Turner, P. P. Wang, I. J. Amster. Anal. Chem. 65, 1746 (1993).

277. S. Guan, M. Wahl, T. Wood, A. Marshall. Anal. Chem. 65, 1753 (1993).

278. G. L. Glish, D. E. Goeringer. Anal. Chem. 56, 2291 (1984).

279. R. J. Beynon, M. K. Doherty, J. M. Pratt, S. J. Gaskell. Nature Meth. 2, 587 (2005).

280. H. M. Rosenstock, M. B. Wallenstein, A. L. Wahrhaftig, H. Eyring. Proc. Natl. Acad. Sci. USA 38, 667 (1952).

281. T. Baercor, P. M. Mayer. J. Am. Soc. Mass Spectrom. 8, 103 (1997).

282. R. E. March, A. W. McMahon, E. T. Allinson, F. A. Londry, R. L. Alfred, J. F. J. Todd, F. Vedel. Int. J. Mass Spectrom. Ion Processes 99, 109 (1990).

283. L. Tang, P. Kebarle. Anal. Chem. 65, 3654 (1993).

284. B. Munson. Int. J. Mass. Spectrom. 200, 243 (2000).

285. A. G. Harrison. Chemical Ionization Mass Spectrometry, CRC Press, Boca Raton (1992).

286. Z. Ouyang, G. Wu, Y. Song, H. Li, W. R. Plass, R. G. Cooks. Anal. Chem. 76, 4595 (2004).

287. J. M. Wilson. Annu. Rep. Prog. Chem. 63, 270 (1966).

288. Y. G. C. Lifshitz, A. Ioffe, J. Laskin, S. Shaik. Int. J. Mass Spectrom. Ion Processes 125, R7 (1993).

289. K. M. Ervin. Chem. Rev. 101, 391 (2001).

290. B. A. Mamyrin, V. I. Karataev, D. V. Shmikk, V. A. Zagulin. Sov. Phys. JETP 37, 45 (1973).

291. J. F. O'Hanlon. A User's Guide to Vacuum Technology, John Wiley, New York (2003).

292. G. L. Glish, D. J. Burinsky. J. Am. Soc. Mass Spectrom. 19, 161 (2008).

293. J. Laskin, J. H. Futrell. Mass Spectrom. Rev. 24, 135 (2005).

294. L. Zandee, R. B. Bernstein. J. Chem. Phys. 71, 1359 (1979).

295. J. D. Fassett, L. J. Moore, J. C. Travis, F. E. Lytle. Int. J. Mass Spectrom. Ion Processes 54, 201 (1983).

296. J. E. Fulford, D.-N. Hoa, R. J. Hughes, R. E. March, R. F. Bonner, G. J. Wong. J. Vac. Sci. Technol. 17, 829 (1980).

297. K. Biemann. Angew. Chem., Int. Ed. Engl. 1, 98 (1962).

298. R. A. Marcus, O. K. Rice. J. Phys. Colloid Chem. 55, 894 (1951).

299. R. A. Marcus. J. Chem. Phys. 20, 359 (1952).

300. S. C. Tucker. Theor. Chem. Acc. 103, 209 (2000).

301. O. K. Rice, H. C. Ramsperger. J. Am. Chem. Soc. 50, 617 (1928).

302. L. S. Kassel. J. Phys. Chem. 32, 225 (1928).

303. E. Schymanski, C. Meinert, M. Meringer, W. Brack. Anal. Chim. Acta 615, 136 (2008).

304. J. Franks. J. Vac. Sci. Technol. 16, 181 (1979).

305. M. H. Lamoree, U. R. Tjaden, J. van der Greef. J. Chromatogr., A 712, 219 (1995).

306. J. F. Anacleto, R. K. Boyd, M. A. Quilliam. J. High Res. Chromatogr. 16, 85 (1993).

(C) 2013, IUPAC

Pure Appl. Chem., Vol. 85, No. 7, pp. 1515-1609, 2013 
307. M. Van Gorkom, R. E. Glick. Int. J. Mass Spectrom. Ion Phys. 4, 203 (1970).

308. A. Benninghoven, B. Hagenhoff, E. Niehuis. Anal. Chem. 65, 630A (1993).

309. H. Oechsner, W. Gerhard. Phys. Lett. A 40, 211 (1972).

310. D. Lipinsky, R. Jede, O. Ganschow, A. Benninghoven. J. Vac. Sci. Technol. 3, 2007 (1985).

311. A. O. Nier. Int. J. Mass Spectrom. Ion Processes 100, 1 (1990).

312. N. G. Adams, D. Smith. Int. J. Mass Spectrom. Ion Phys. 21, 349 (1976).

313. J. T. Watson. Introduction to Mass Spectrometry, Lippincott-Raven, Philadelphia (1997).

314. E. de Hoffmann. J. Mass Spectrom. 31, 129 (1996).

315. S. Ghaderi, P. S. Kulkarni, E. B. Ledford, C. L. Wilkins, M. L. Gross. Anal. Chem. 53, 428 (1981).

316. S. J. Gaskell. J. Mass Spectrom. 32, 677 (1997).

317. M. L. Fournier, J. M. Gilmore, S. A. Martin-Brown, M. P. Washburn. Chem. Rev. 107, 3654 (2007)

318. D. E. Games. Chem. Phys. Lipids 21, 389 (1978).

319. A. Hirabayashi, M. Sakairi, H. Koizumi. Anal. Chem. 66, 4557 (1994).

320. J. F. J. Todd, R. M. Waldren, R. E. Mather. Int. J. Mass Spectrom. Ion Phys. 34, 325 (1980).

321. R. M. Smith. Understanding Mass Spectra, Wiley-Interscience, Hoboken (2004).

322. M. Sakairi, H. Kambara. Anal. Chem. 61, 1159 (1989).

323. H. Jiang, A. M. English. J. Proteome Res. 1, 345 (2002).

324. S. E. Ong, B. Blagoev, I. Kratchmarova, D. B. Kristensen, H. Steen, A. Pandey, M. Mann. Mol. Cell Proteomics 1, 376 (2002).

325. H. Zhu, S. Pan, S. Gu, E. M. Bradbury, X. Chen. Rapid Commun. Mass Spectrom. 16, 2115 (2002).

326. L. Anderson, C. L. Hunter. Mol. Cell Proteomics 5, 573 (2006).

327. M. Oron. Nucl. Instrum. Meth. 139, 235 (1976).

328. D. P. Stevenson. Disc. Faraday Soc. 10, 35 (1951).

329. M. M. Bursey, E. S. Wolfe. Org. Mass Spectrom. 1, 543 (1968).

330. A. G. Harrison, C. D. Finney, J. A. Sherk. Org. Mass Spectrom. 5, 1313 (1971).

331. S. Guan, A. G. Marshall. Int. J. Mass Spectrom. Ion Processes 157/158, 5 (1996).

332. L. G. Randall, A. L. Wahrhaftig. Rev. Sci. Instrum. 52, 1283 (1981).

333. A. M. Dattelbaum, S. Iyer. Exp. Rev. Proteomics 153 (2006).

334. T. W. Hutchens, T. T. Yip. Rapid Commun. Mass Spectrom. 7, 576 (1993).

335. T. C. W. Poon. Exp. Rev. Proteomics 4, 51 (2007).

336. R. G. Cooks, D. T. Terwilliger, T. Ast, J. H. Beynon, T. Keough. J. Am. Chem. Soc. 97, 1583 (1995).

337. V. Grill, R. Wörgötter, J. H. Futrell, T. D. Mark. Z. Phys. D 40, 111 (1997).

338. A. Fischer, P. Werner. Fresenius' J. Anal. Chem. 368, 424 (2000).

339. J. W. Gauthier, T. R. Trautman, D. B. Jacobson. Anal. Chim. Acta 246, 211 (1991).

340. J. V. Johnson, R. A. Yost, P. E. Kelley, D. C. Bradford. Anal. Chem. 62, 2162 (1990).

341. G. Taylor. Proc. Roy. Soc. Lond. Ser. A 280, 383 (1964).

342. S. J. Gaskell. J. Mass Spectrom. 32, 677 (1997).

343. C. R. Blakley, J. J. Carmody, M. L. Vestal. Anal. Chem. 52, 1636 (1980).

344. C. R. Blakley, M. L. Vestal. Anal. Chem. 55, 750 (1983).

345. O. S. Ovchinnikova, G. J. Van Berkel. Rapid Commun. Mass Spectrom. 24, 1721 (2010).

346. W. C. Wiley, I. H. McLaren. Rev. Sci. Instrum. 26, 1150 (1955).

347. S. K. Sze, Y. Ge, H. Oh, F. W. McLafferty. Proc. Natl. Acad. Sci. USA 99, 1774 (2002).

348. N. L. Kelleher. Anal. Chem. 76, 197A (2004).

349. S. A. Lammert, A. A. Rockwood, M. Wang, M. L. Lee, E. D. Lee, S. E. Tolley, J. R. Oliphant, J. L. Jones, R. W. Waite. J. Am. Soc. Mass Spectrom. 17, 916 (2006).

350. T. Mizuno, N. Abe, H. Teshima, E. Yamauchi, Y. Itagaki, I. Matsumoto, T. Kuhara, T. Shinka. Biomed. Mass Spectrom. 8, 593 (1981). 
351. J. A. Olivares, N. T. Nguyen, C. R. Yonker, R. D. Smith. Anal. Chem. 59, 1230 (1997).

352. J. T. Watson. Annu. Rev. Pharmacol. 13, 391 (1973).

353. R. M. Smith. Understanding Mass Spectra: A Basic Approach, John Wiley, New York (2004).

354. R. D. Voyksner, T. Pack. Rapid Commun. Mass Spectrom. 5, 263 (1991).

355. P. J. Robinson, K. A. Holbrook. Unimolecular Reactions, John Wiley, New York (1972).

356. C. F. Robinson. Rev. Sci. Instrum. 27, 88 (1956).

357. T. Staaf, C. Östman. J. Environ. Monitor. 7, 344 (2005).

358. A. G. Brenton, R. P. Morgan, J. H. Beynon. Ann. Rev. Phys. Chem. 30, 51 (1979).

359. H. H. Rose. Sci. Technol. Adv. Mater. 9, 014107 (2008).

Republication or reproduction of this report or its storage and/or dissemination by electronic means is permitted without the need for formal IUPAC permission on condition that an acknowledgment, with full reference to the source, along with use of the copyright symbol $\odot$, the name IUPAC, and the year of publication, are prominently visible. Publication of a translation into another language is subject to the additional condition of prior approval from the relevant IUPAC National Adhering Organization. 